Supporting Information for:

\title{
NaH Promoted One-Pot Tandem Reactions of 3-(1-Alkynyl) \\ Chromones to Form 2-Nitrogen-Substituted Xanthones
}

\author{
Wen-Di Duan, ${ }^{\dagger}+$ Yu-Fang Zhang, ${ }^{\dagger} *$ and Youhong $\mathrm{Hu}^{* \dagger *}$ \\ 'State Key Laboratory of Drug Research, Shanghai Institute of Materia Medica, Chinese Academy \\ of Sciences, 555 Zuchongzhi Road, Shanghai 201203, China. \\ tUniversity of Chinese Academy of Sciences, No.19A Yuquan Road, Beijing 100049, China.
}

Table of Contents:

1. Initial attempts in investigation of isocyanoacetates and 3-(1-alkynyl)-chromones..........S2

2. NMR Spectra..... $\mathrm{S} 3-\mathrm{S} 19$

3. X-ray Crystallography data $\mathrm{S} 20-\mathrm{S} 21$ 
1. Initial attempts in investigation of isocyanoacetates and 3-(1-alkynyl)-chromones

Table S1: Initial attempts in investigation of isocyanoacetates and 3-(1-alkynyl)-chromones ${ }^{\mathrm{a}}$

\begin{tabular}{|c|c|c|}
\hline Entry & Condition & Results \\
\hline 1 & $\mathrm{Ag}_{2} \mathrm{O}, \mathrm{Et}_{3} \mathrm{~N}, \mathrm{rt}$ & NR, Substrate Recovered \\
\hline 2 & DMF, DBU, AgOTf, $\mathrm{Sc}(\mathrm{OTf})_{3}, 50^{\circ} \mathrm{C}$ & Messy \\
\hline 3 & $\mathrm{DMF}, \mathrm{CuCl}, \mathrm{Cs}_{2} \mathrm{CO}_{3}, \mathrm{Ar}, 100^{\circ} \mathrm{C}$ & Messy \\
\hline 4 & $\mathrm{CH}_{3} \mathrm{CN}, \mathrm{CuCl}, \mathrm{Ar}, 50^{\circ} \mathrm{C}$ & Furocoumarin $\mathbf{A}^{\mathrm{b}}$ \\
\hline 5 & $\mathrm{MeCN}, \mathrm{CuCl}, \mathrm{Cs}_{2} \mathrm{CO}_{3}, \mathrm{Ar}, 50^{\circ} \mathrm{C}$ & $3 \mathbf{a}$ \\
\hline 6 & $\mathrm{MeCN}, \mathrm{CuCl}, \mathrm{Cs}_{2} \mathrm{CO}_{3}, \mathrm{Ar}, \mathrm{rt}$ & $\mathbf{3 a}$ \\
\hline 7 & $\mathrm{MeCN}, \mathrm{CuCl}, \mathrm{Cs}_{2} \mathrm{CO}_{3}$, Ar, reflux & $3 \mathbf{a}$ \\
\hline 8 & $\mathrm{NMP}, \mathrm{Ag}_{2} \mathrm{O}, \mathrm{K}_{2} \mathrm{CO}_{3}, \mathrm{MW}, 130^{\circ} \mathrm{C}$ & Messy \\
\hline 9 & $\mathrm{NMP}, \mathrm{AgOAc}, \mathrm{K}_{2} \mathrm{CO}_{3}, \mathrm{MW}, 130^{\circ} \mathrm{C}$ & $\mathbf{2 a}+\mathbf{3 a}$ \\
\hline
\end{tabular}

aThe reaction were carried out with combinations of 0.1 eq base and 0.01 eq transition metal salt. ${ }^{b}$ The furocoumarin compound was found in the experiment following the route in this literature: (1) G. Cheng, Y. Hu. Chem. Commun. 2007, 31, 3285-3287

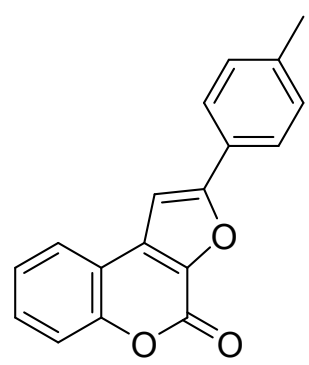

A

Figure S1: Stucture of Furocoumarin A 
2.NMR Spectra

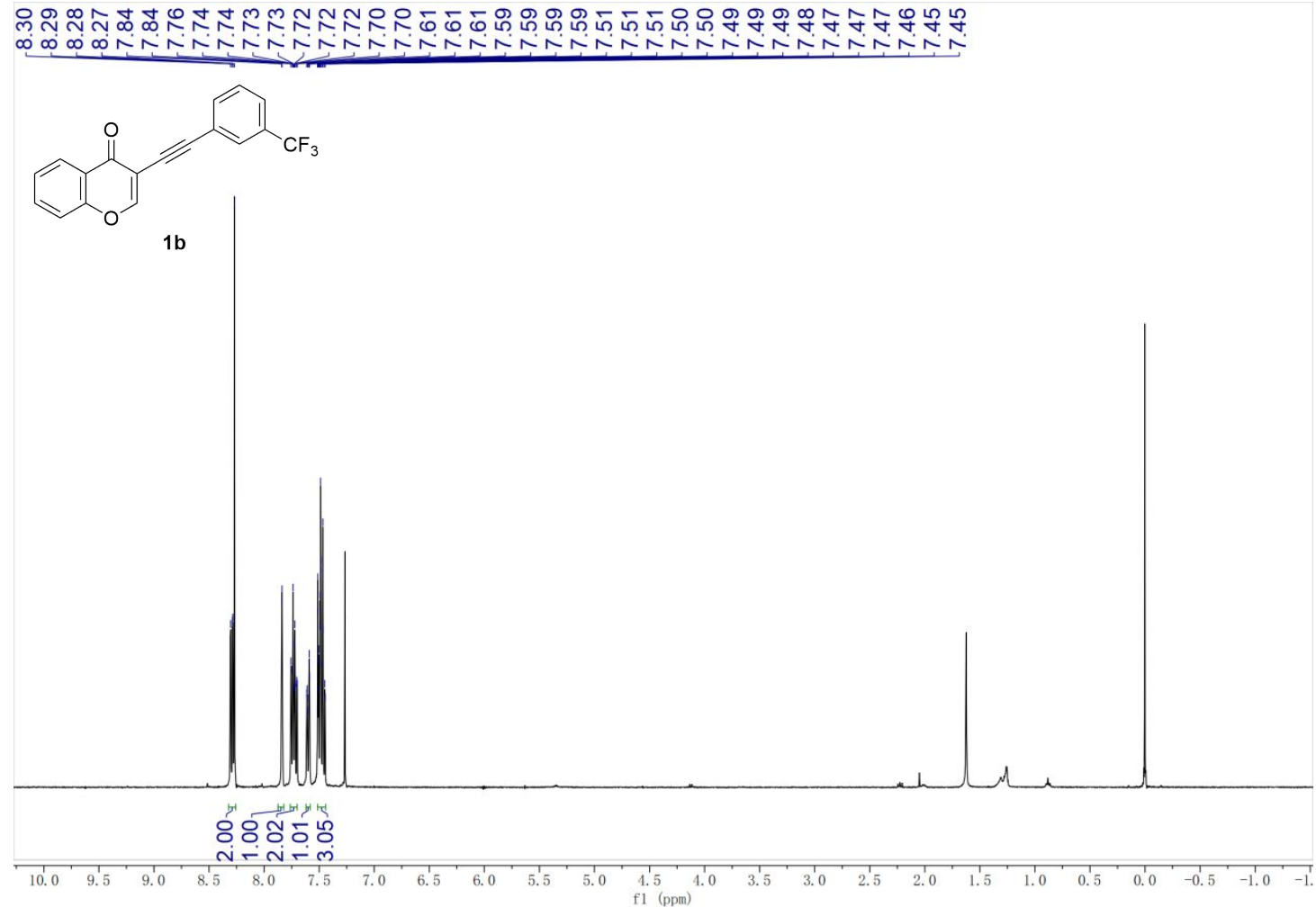

Figure S2: ${ }^{1} \mathrm{H}$ NMR Spectrum of Compound $\mathbf{1 b}\left(400 \mathrm{MHz}, \mathrm{CDCl}_{3}\right)$
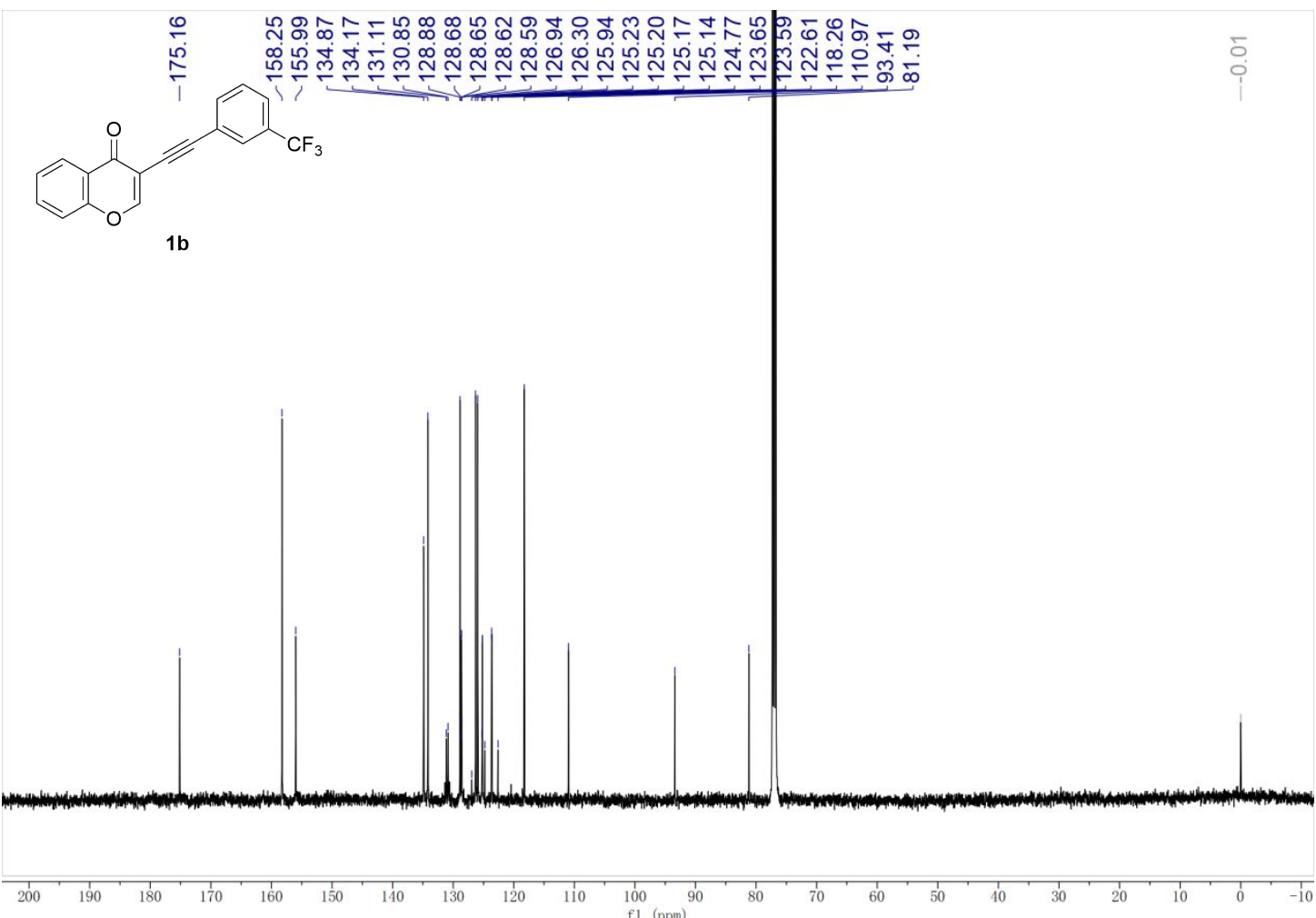

Figure S3: ${ }^{13} \mathrm{C}$ NMR Spectrum of Compound $\mathbf{1 b}\left(125 \mathrm{MHz}, \mathrm{CDCl}_{3}\right)$ 


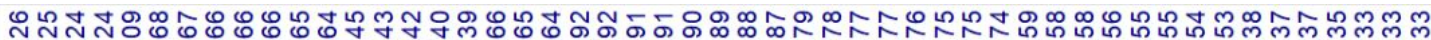

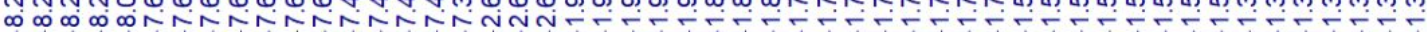<smiles>O=c1c(C#CC2CCCCC2)coc2ccccc12</smiles>
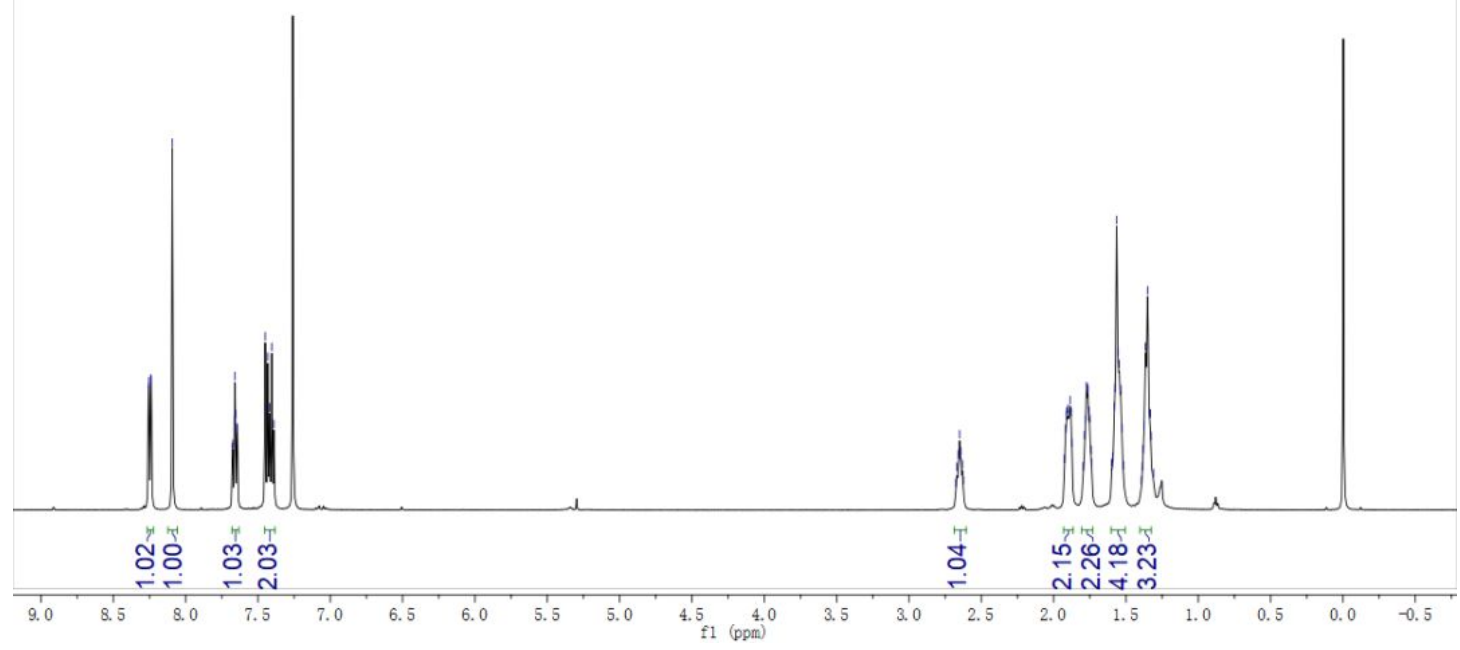

Figure S4: ${ }^{1} \mathrm{H}$ NMR Spectrum of Compound $1 \mathbf{n}\left(500 \mathrm{MHz}, \mathrm{CDCl}_{3}\right)$

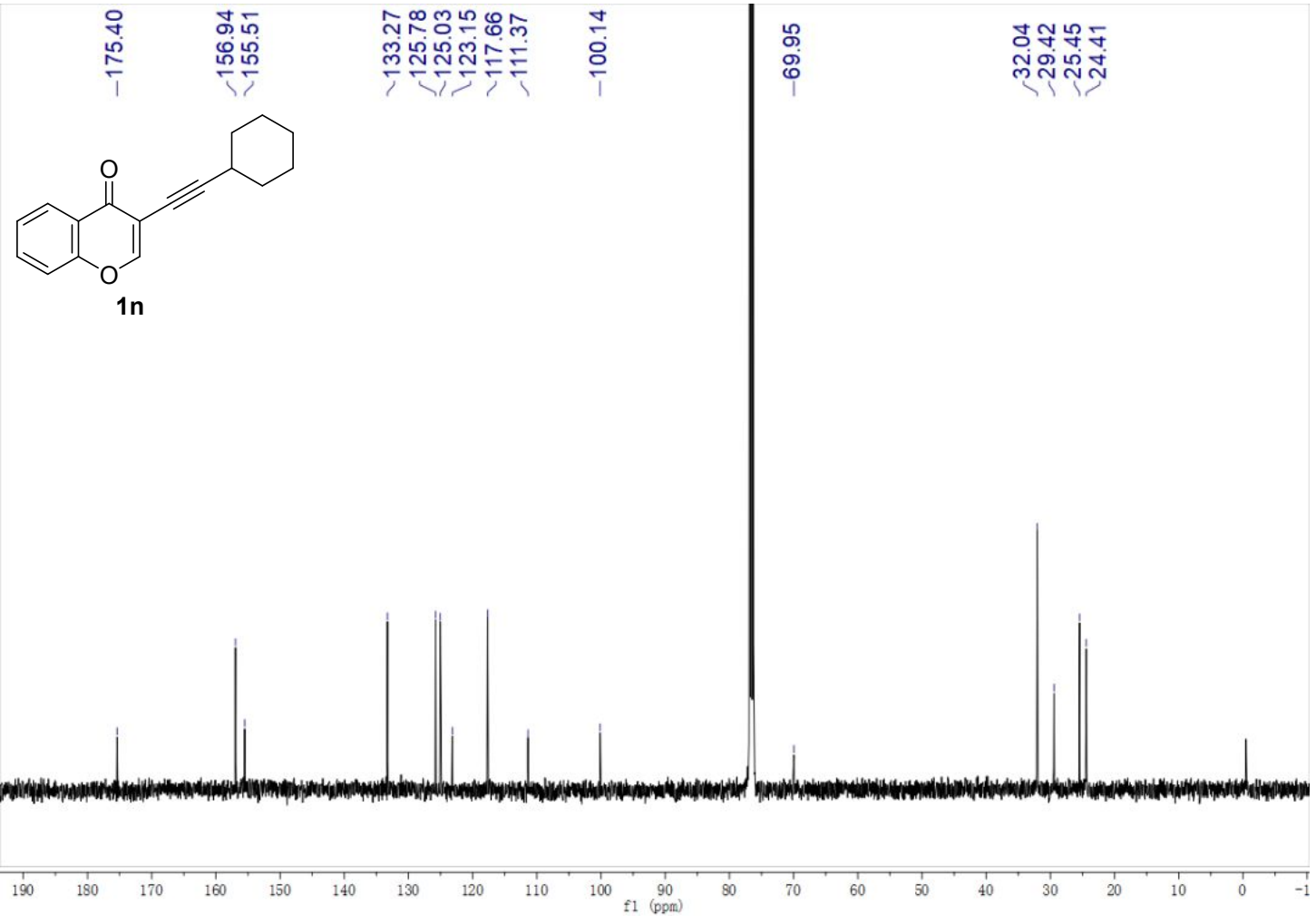

Figure S5: ${ }^{13} \mathrm{C}$ NMR Spectrum of Compound 1 n(125 MHz, $\left.\mathrm{CDCl}_{3}\right)$ 


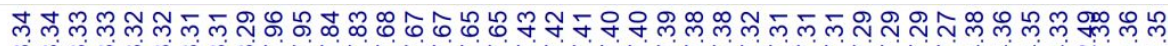

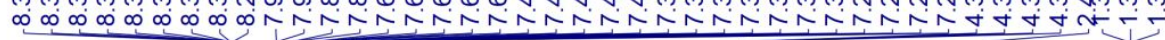

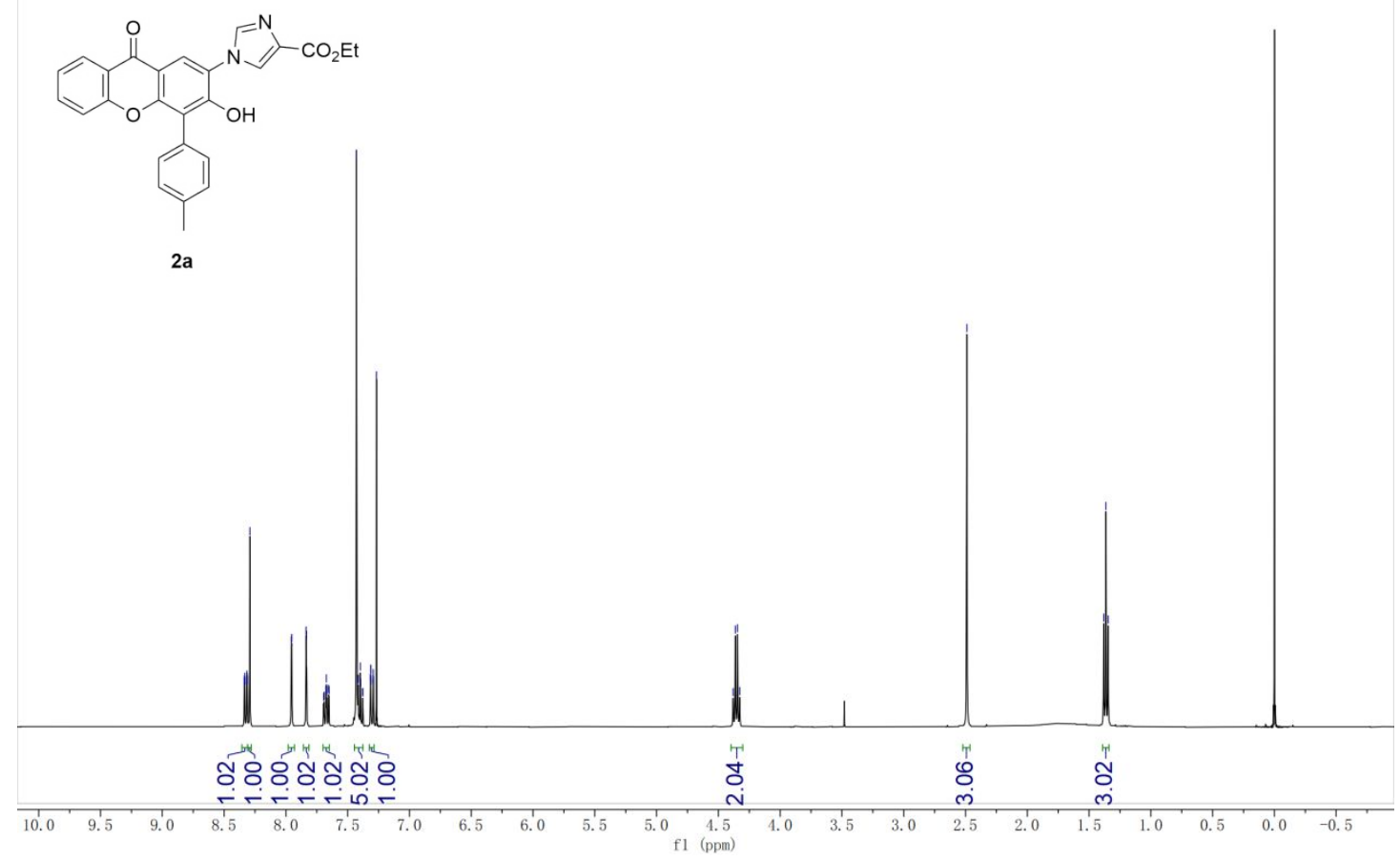

Figure S6: ${ }^{1} \mathrm{H}$ NMR Spectrum of Compound $\mathbf{2 a}\left(400 \mathrm{MHz}, \mathrm{CDCl}_{3}\right)$

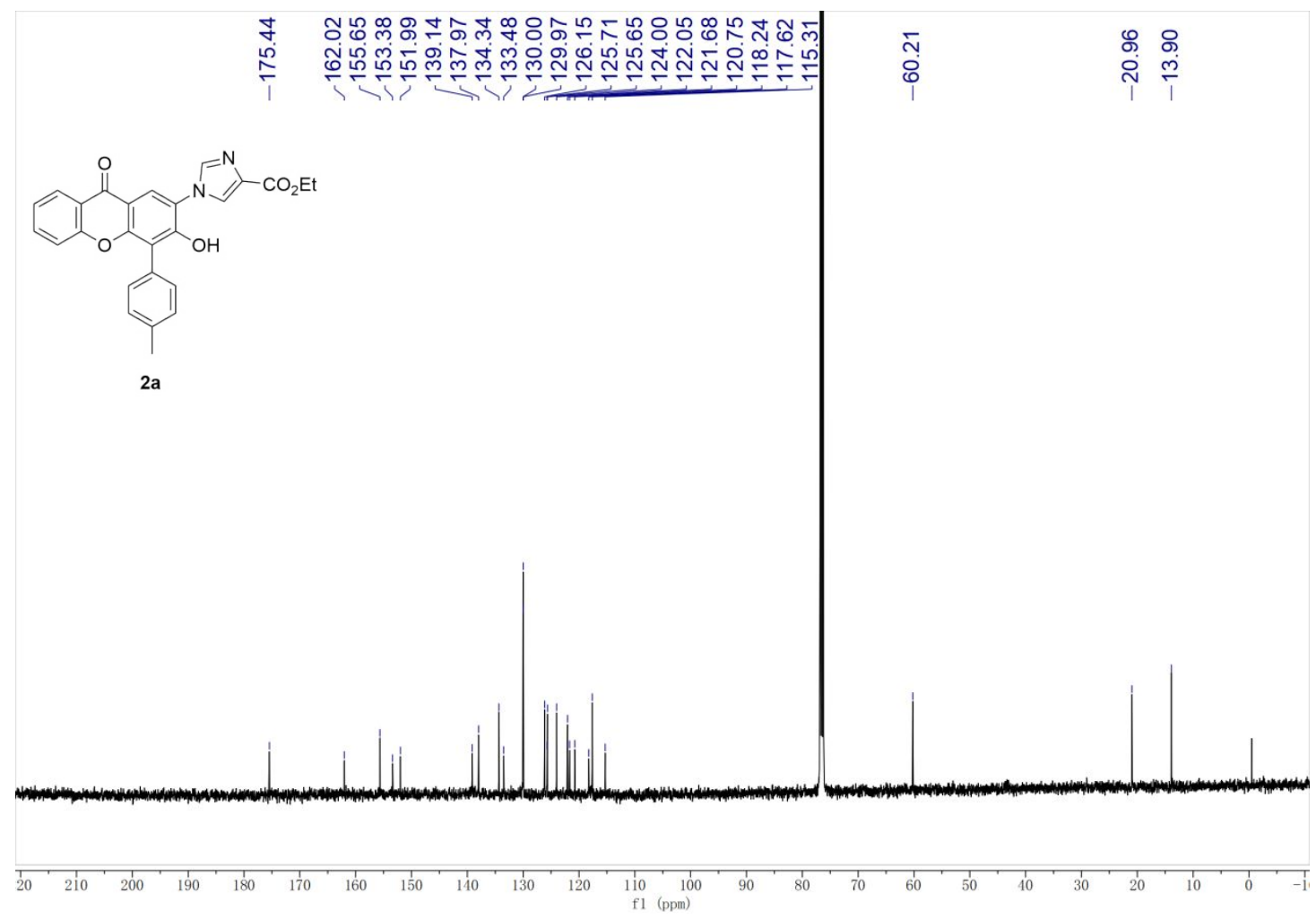

Figure S7: ${ }^{13} \mathrm{C}$ NMR Spectrum of Compound $\mathbf{2 a}\left(125 \mathrm{MHz}, \mathrm{CDCl}_{3}\right)$ 


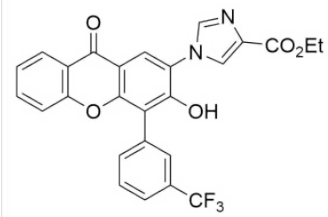

$2 b$
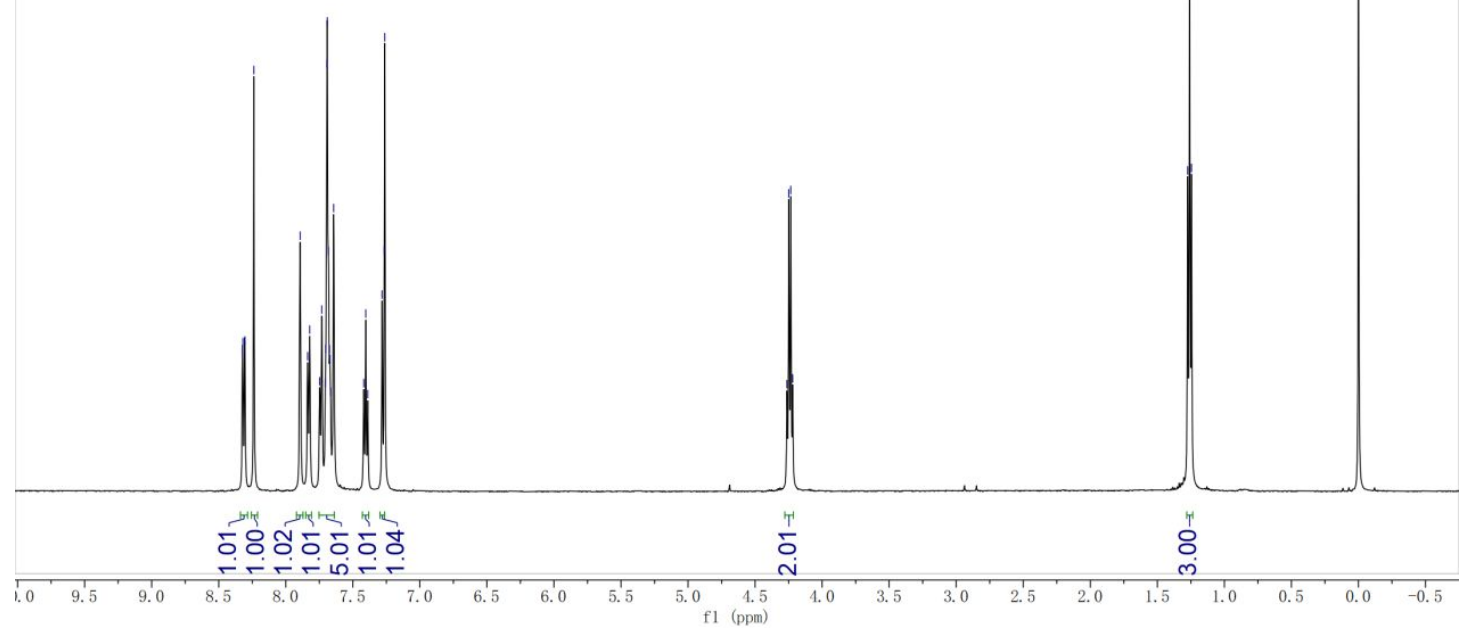

Figure S8: ${ }^{1} \mathrm{H}$ NMR Spectrum of Compound $\mathbf{2 b}\left(500 \mathrm{MHz}, \mathrm{CDCl}_{3}\right)$

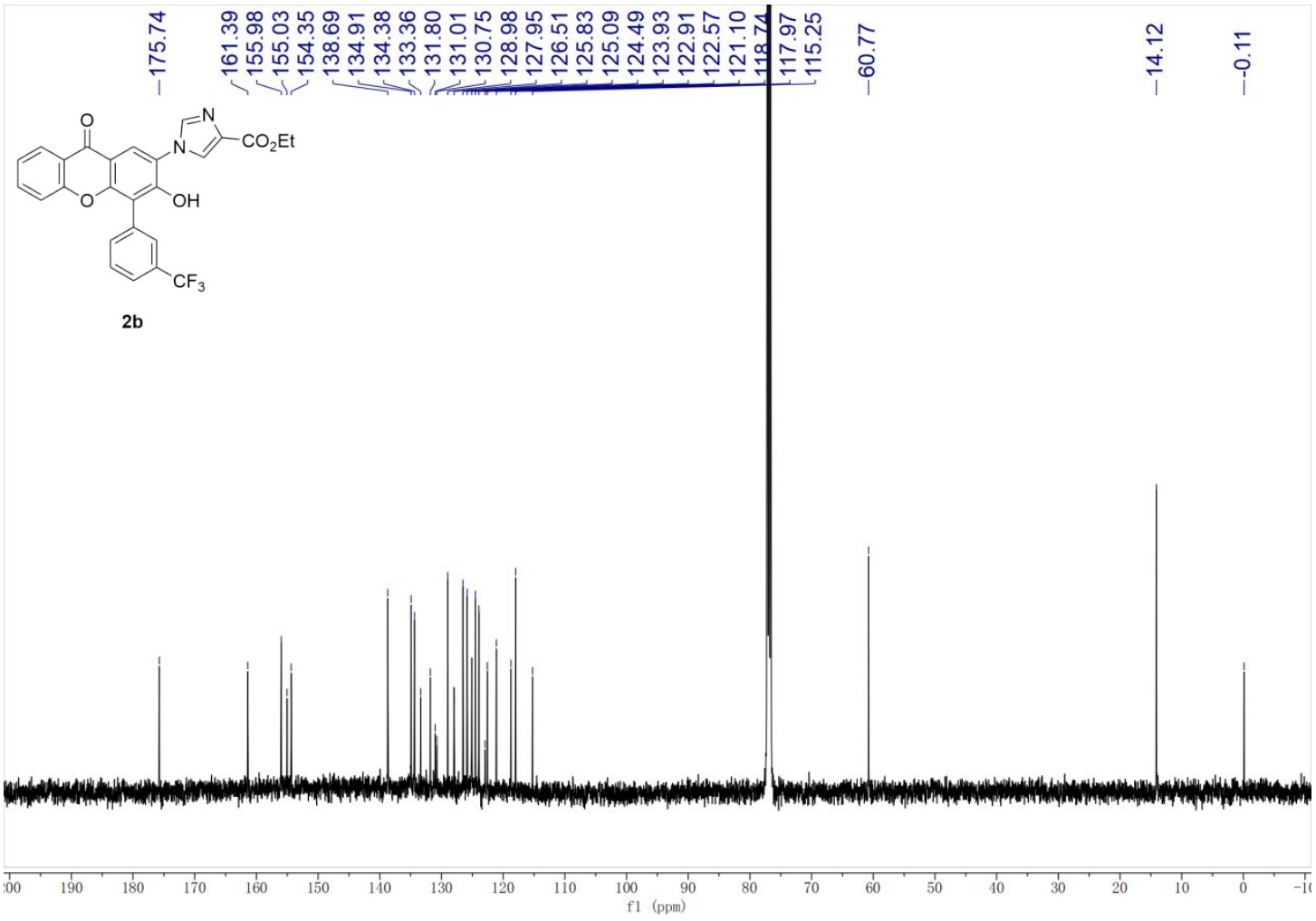

Figure S9: ${ }^{13} \mathrm{C}$ NMR Spectrum of Compound $\mathbf{2 b}\left(125 \mathrm{MHz}, \mathrm{CDCl}_{3}\right)$ 


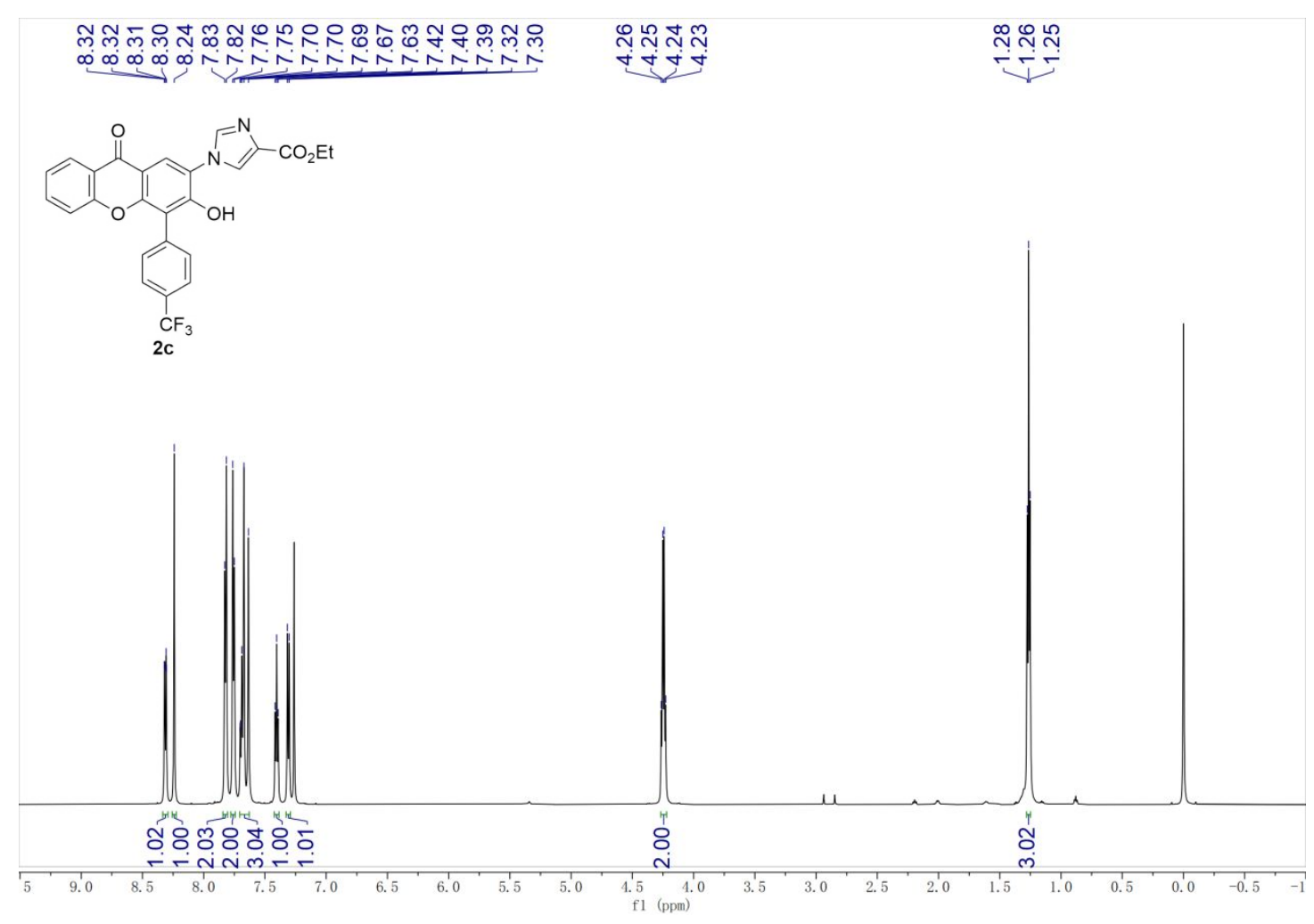

Figure S10: ${ }^{1} \mathrm{H}$ NMR Spectrum of Compound $2 \mathbf{c}\left(600 \mathrm{MHz}, \mathrm{CDCl}_{3}\right)$

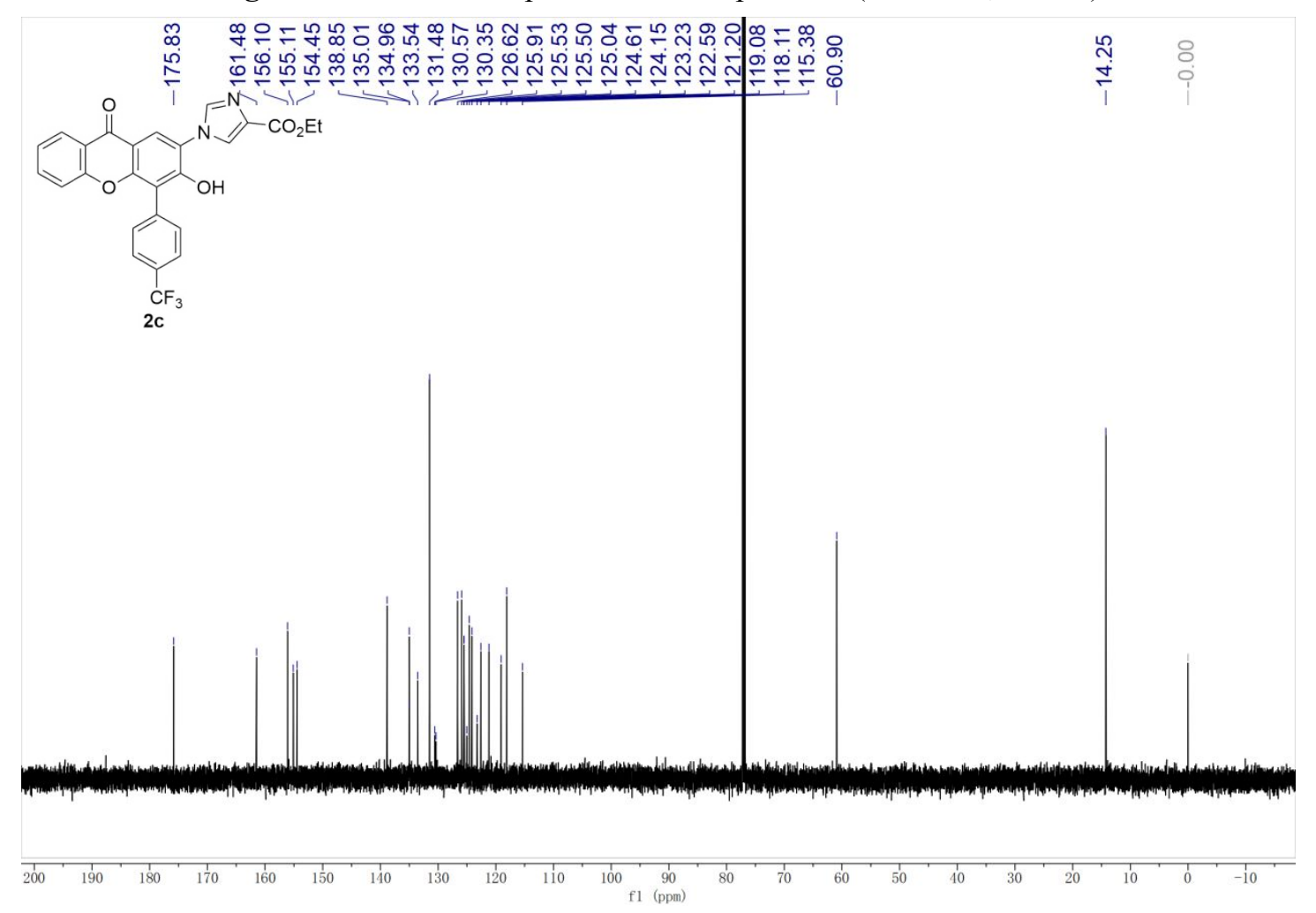

Figure S11: ${ }^{13} \mathrm{C}$ NMR Spectrum of Compound $2 \mathbf{c}\left(150 \mathrm{MHz}, \mathrm{CDCl}_{3}\right)$ 


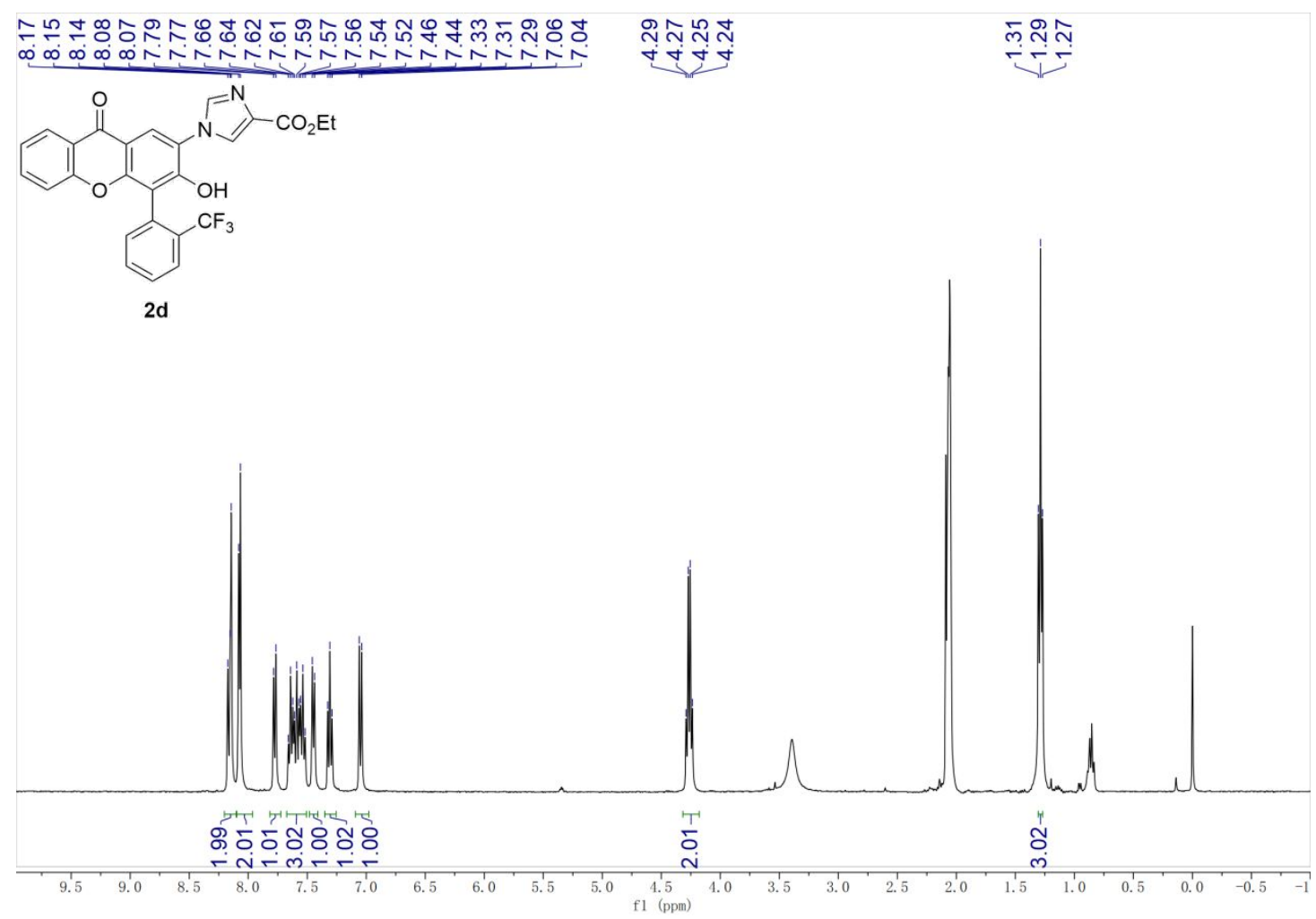

Figure S12: ${ }^{1} \mathrm{H}$ NMR Spectrum of Compound 2d(400 MHz, Acetone- $\left.d_{6}\right)$

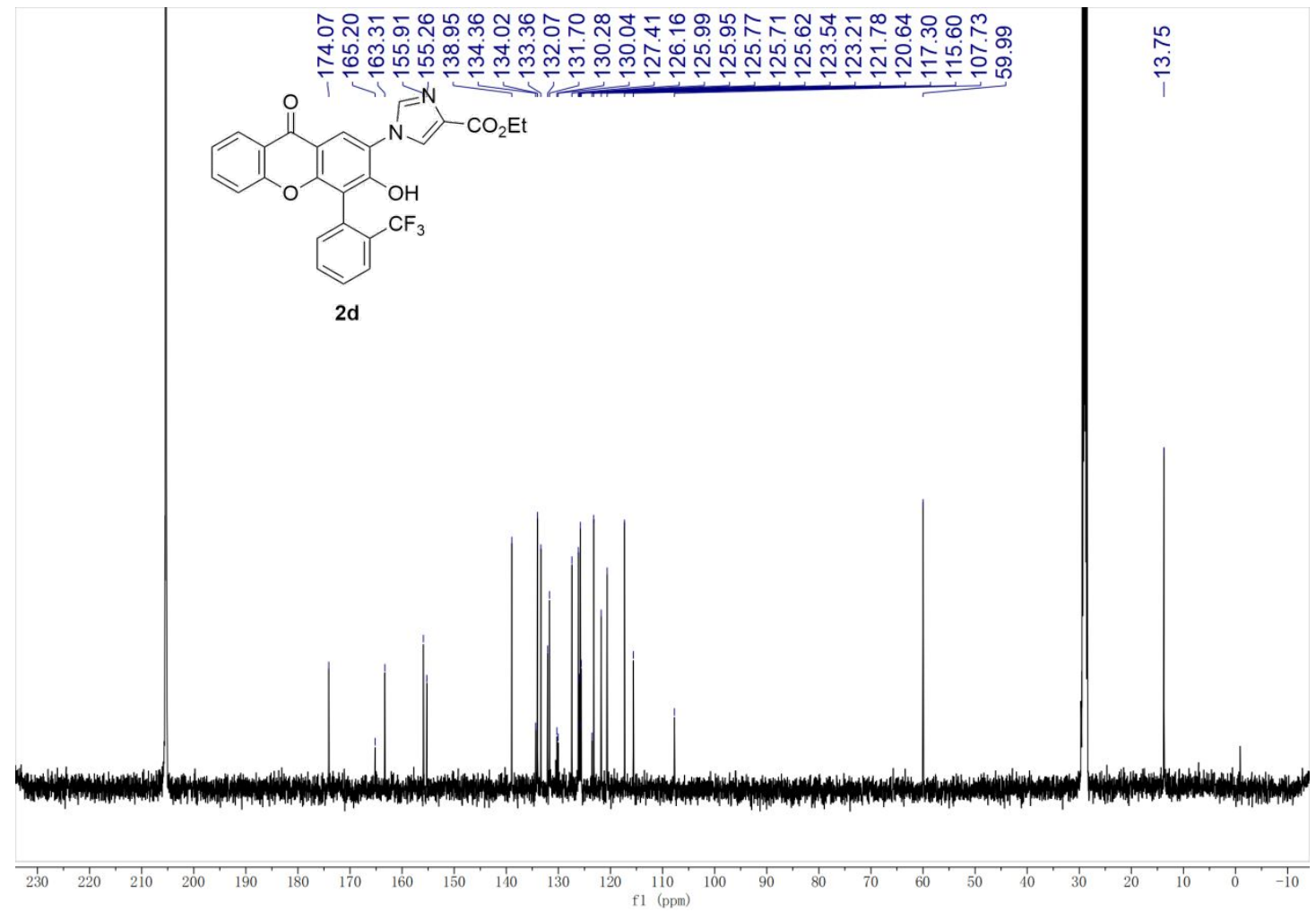

Figure S13: ${ }^{1} \mathrm{H}$ NMR Spectrum of Compound 2d(125 MHz, Acetone- $\left.d_{6}\right)$ 


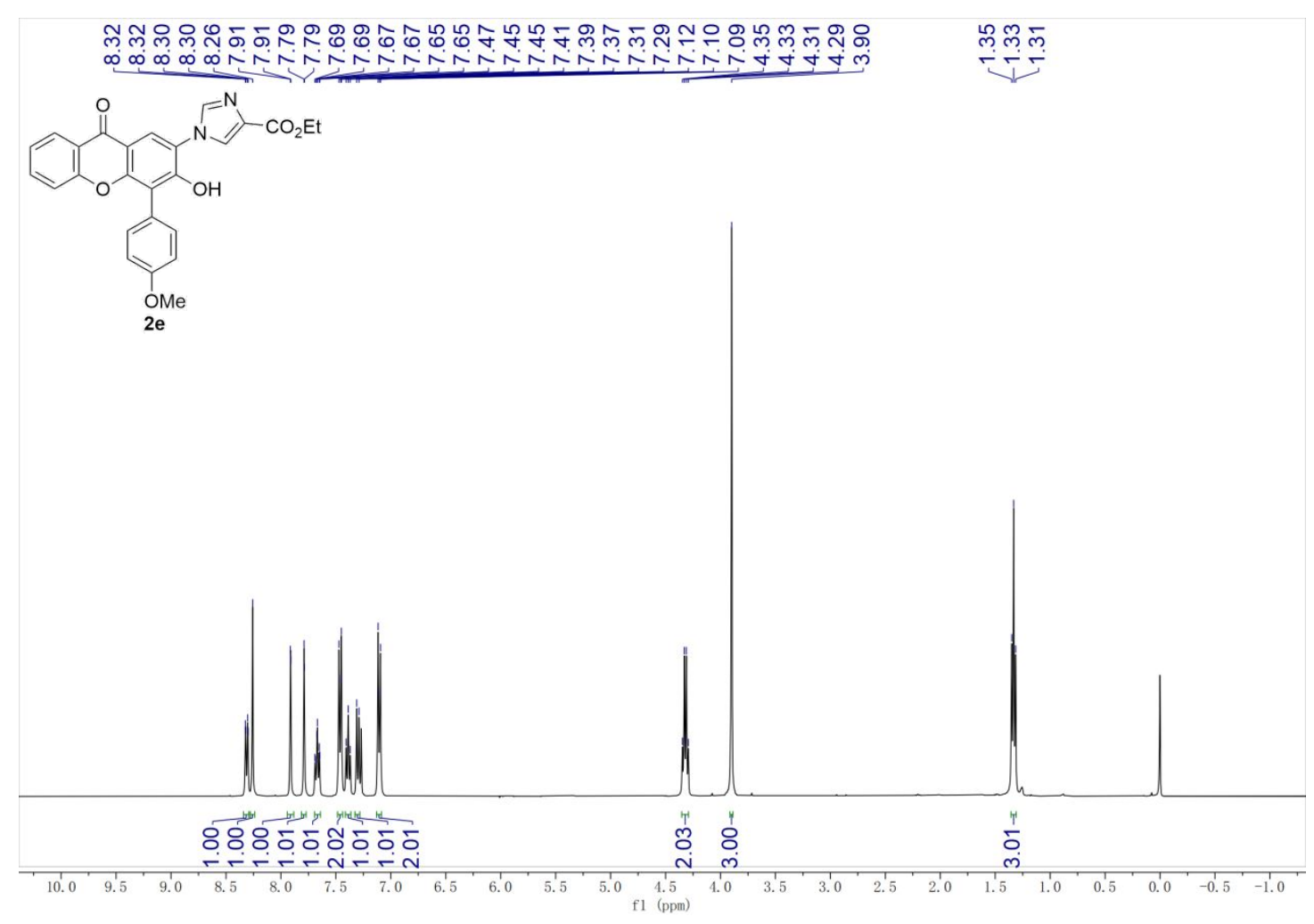

Figure S14: ${ }^{1} \mathrm{H}$ NMR Spectrum of Compound $2 \mathbf{e}\left(400 \mathrm{MHz}, \mathrm{CDCl}_{3}\right)$

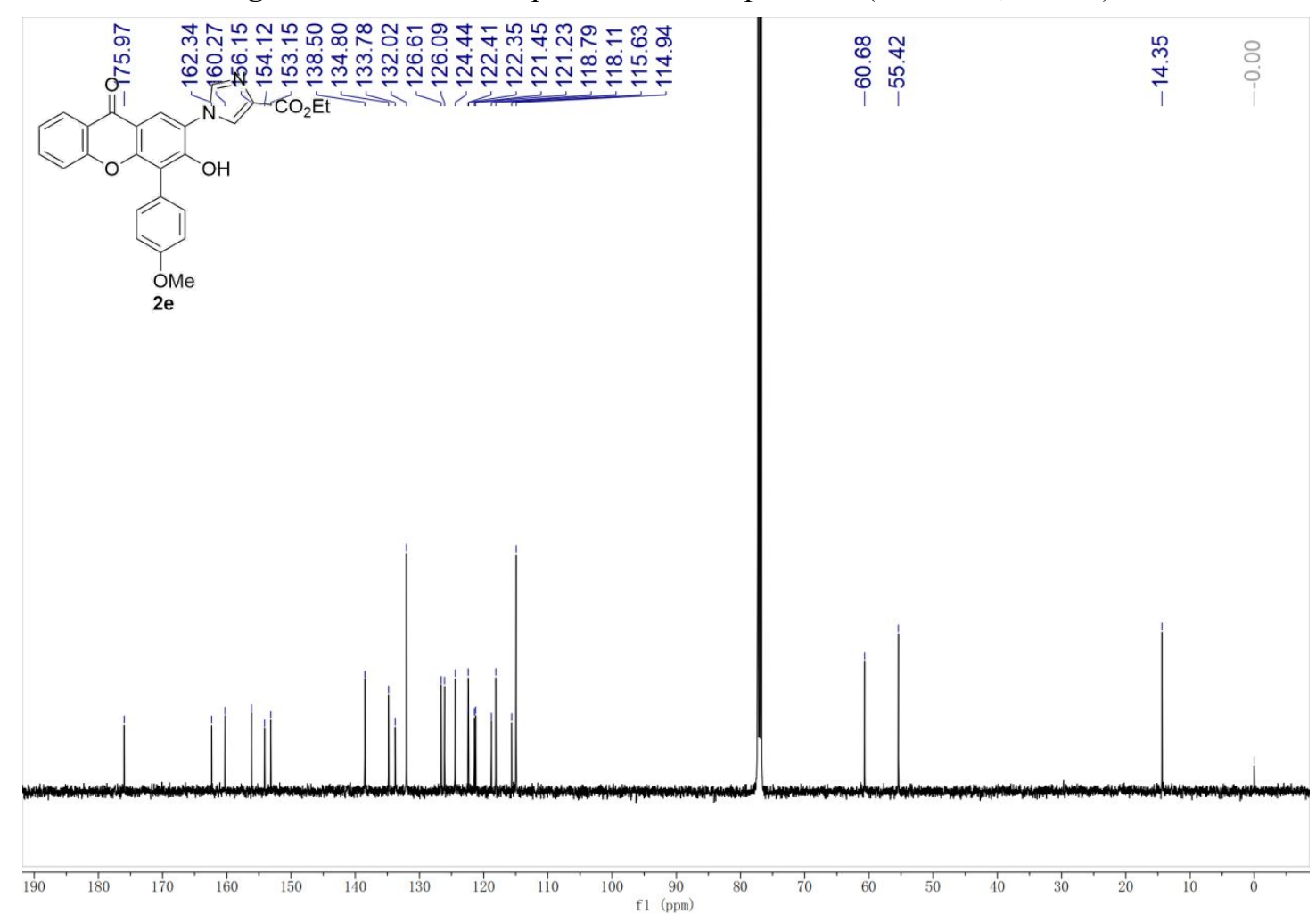

Figure S15: ${ }^{13} \mathrm{C}$ NMR Spectrum of Compound $2 \mathrm{e}\left(125 \mathrm{MHz}, \mathrm{CDCl}_{3}\right)$ 


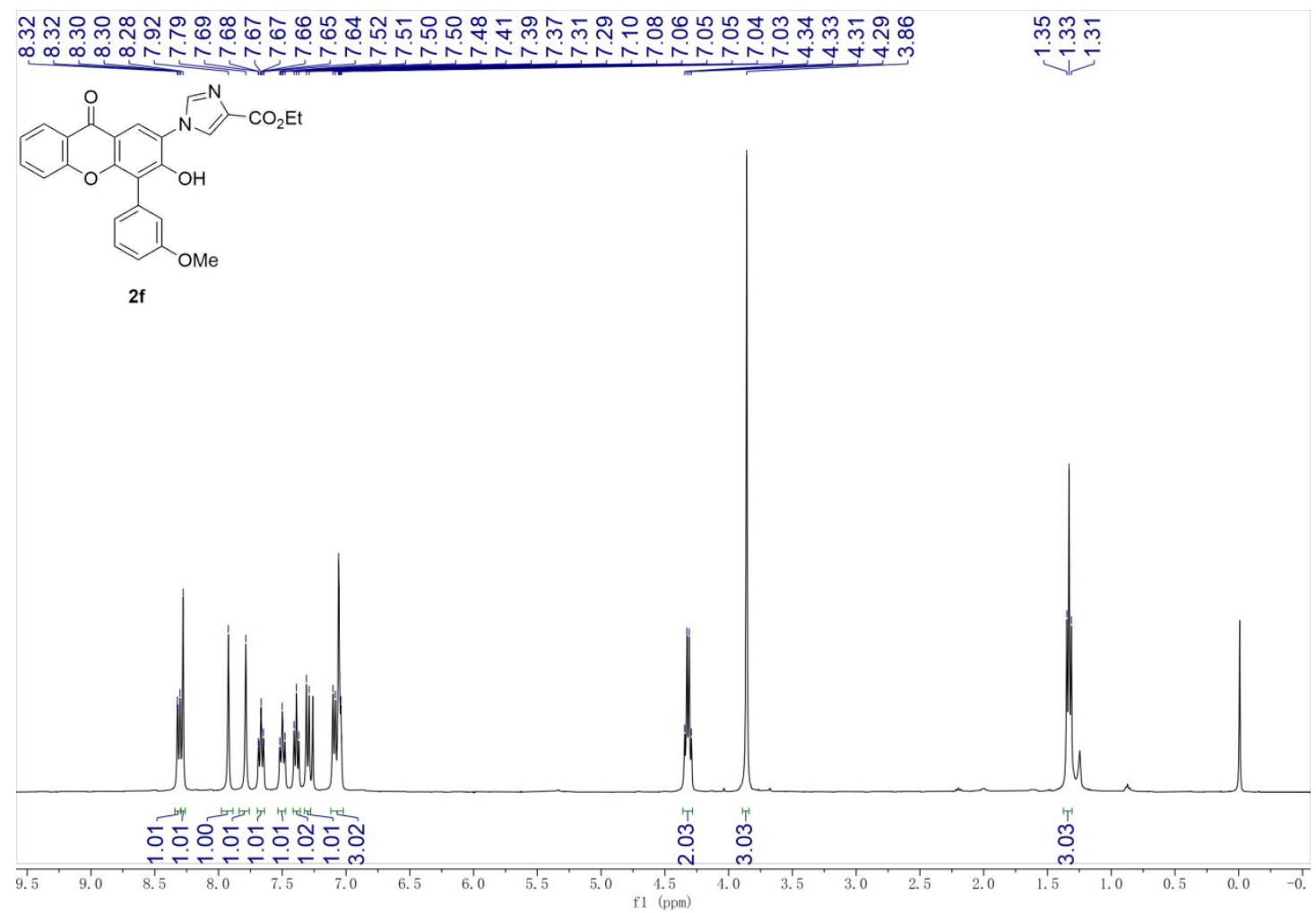

Figure S16: ${ }^{1} \mathrm{H}$ NMR Spectrum of Compound $2 \mathbf{f}\left(400 \mathrm{MHz}, \mathrm{CDCl}_{3}\right)$

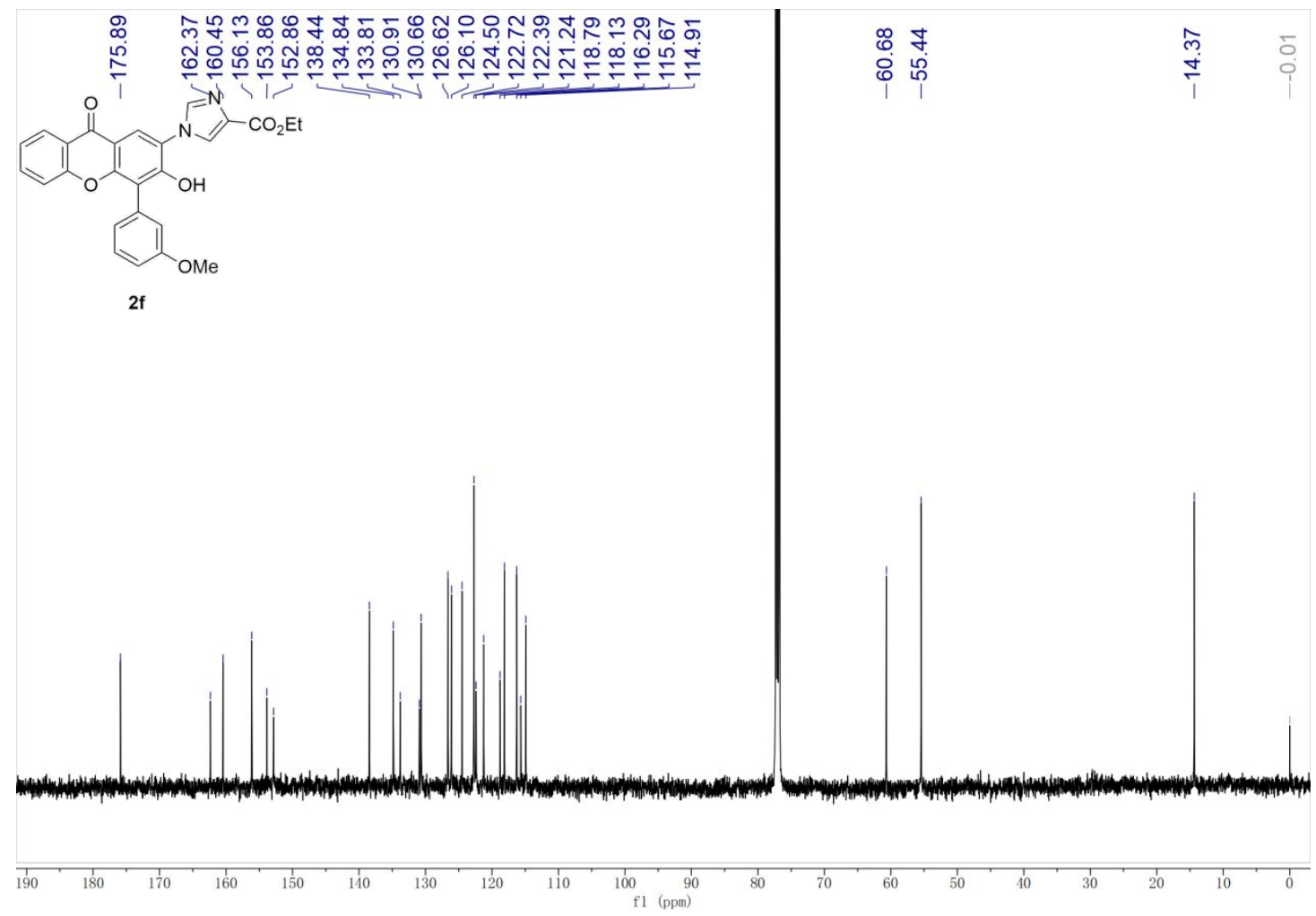

Figure S17: ${ }^{13} \mathrm{C}$ NMR Spectrum of Compound $\mathbf{2} \mathbf{f}\left(125 \mathrm{MHz}, \mathrm{CDCl}_{3}\right)$ 


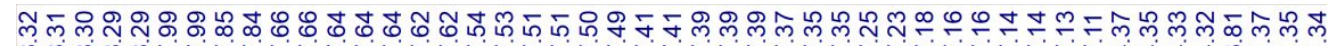

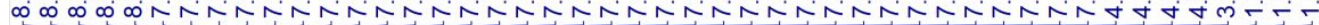<smiles>CCOc1cnn(-c2cc3c(=O)c4ccccc4oc3c(-c3ccccc3OC)c2O)c1</smiles>

$2 \mathrm{~g}$

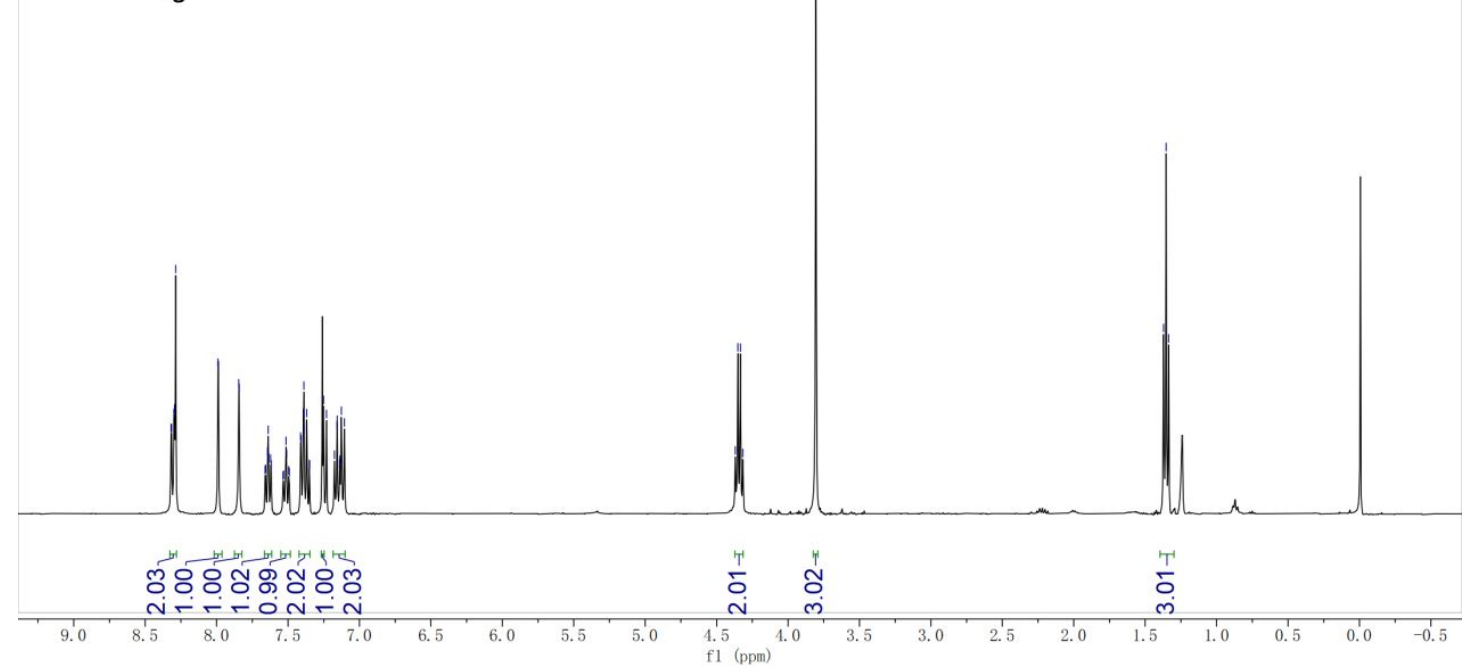

Figure S18: ${ }^{1} \mathrm{H}$ NMR Spectrum of Compound $2 \mathbf{g}\left(400 \mathrm{MHz}, \mathrm{CDCl}_{3}\right)$

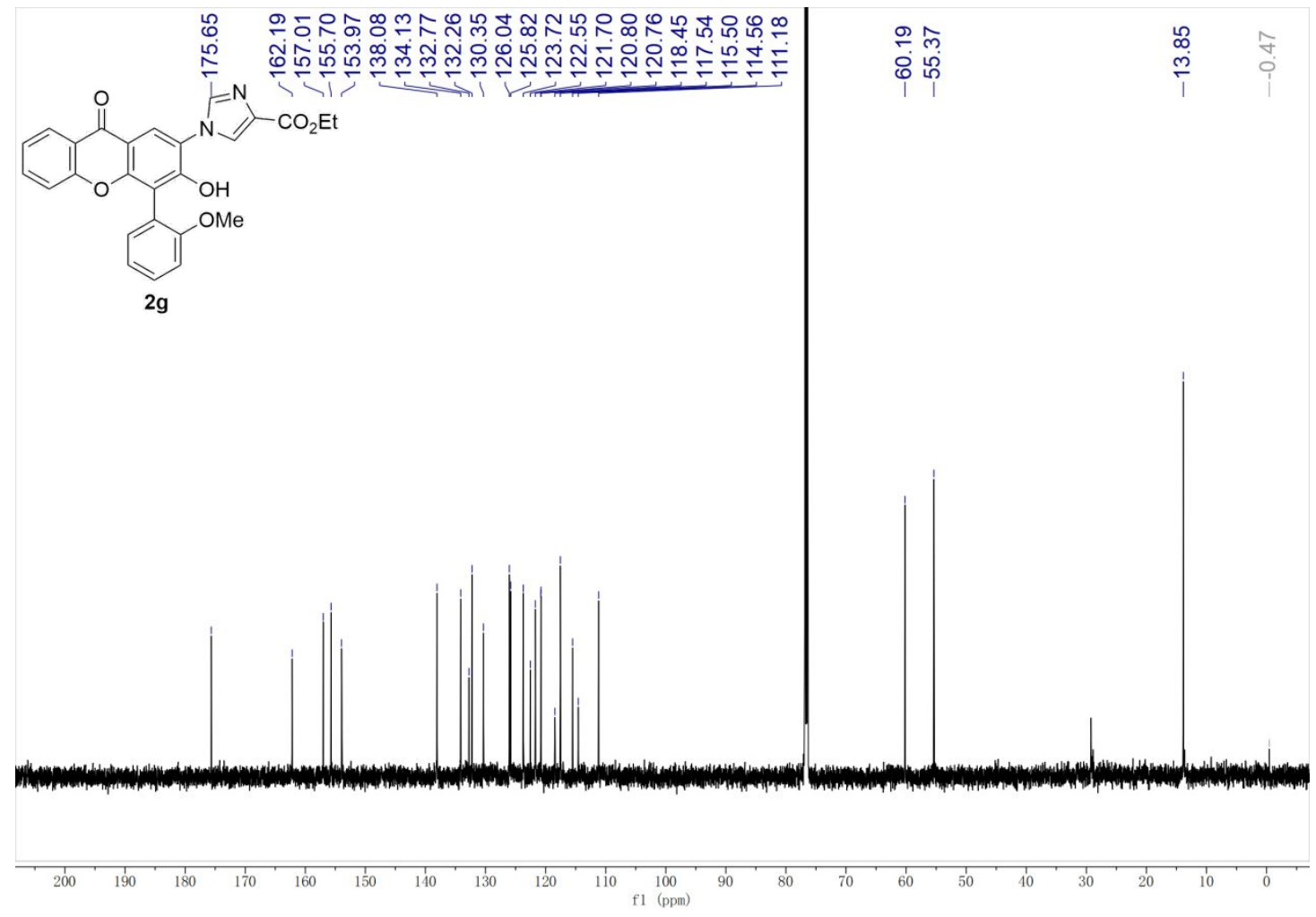

Figure S19: ${ }^{13} \mathrm{C}$ NMR Spectrum of Compound $2 \mathbf{g}\left(150 \mathrm{MHz}, \mathrm{CDCl}_{3}\right)$ 


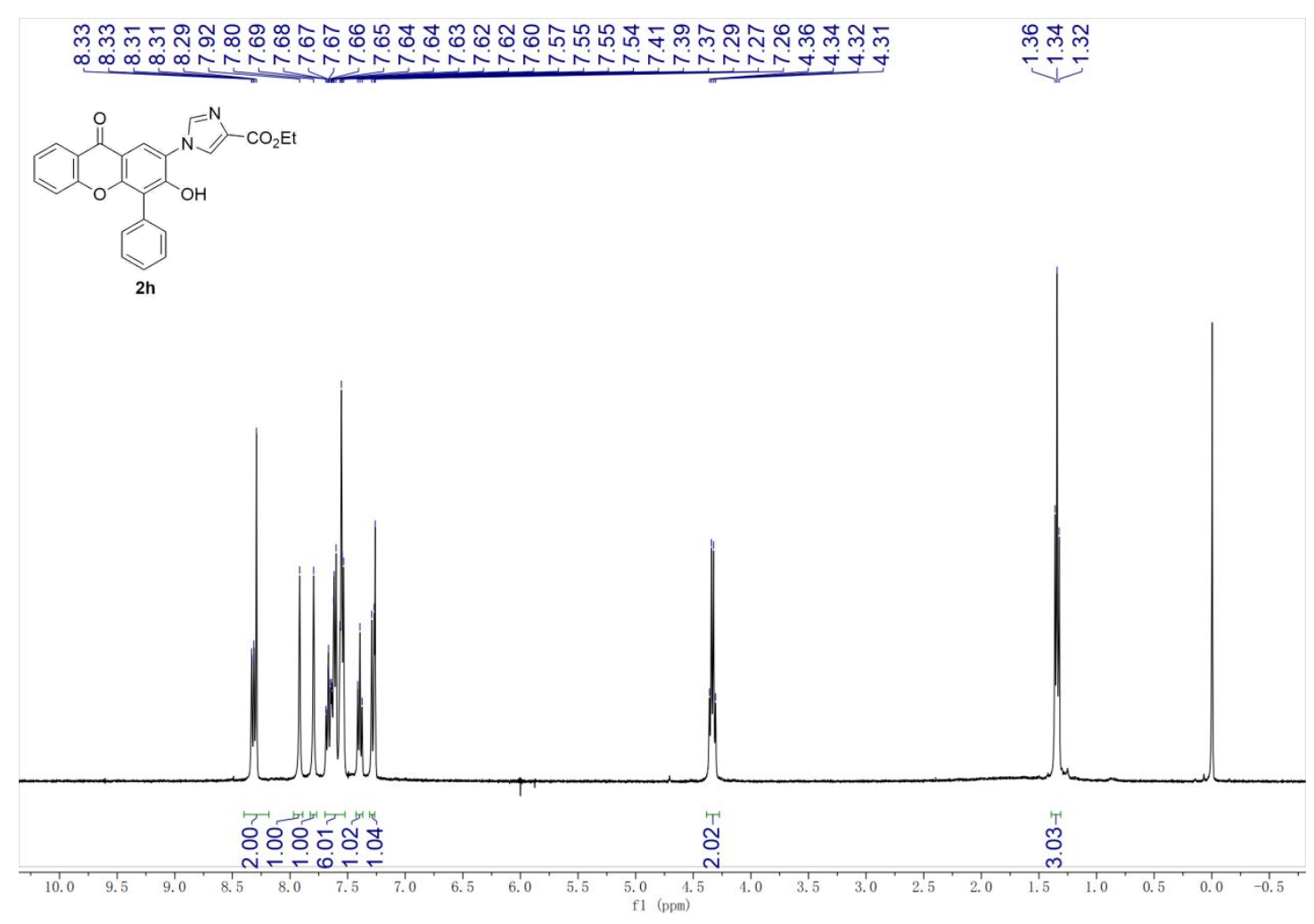

Figure S20: ${ }^{1} \mathrm{H}$ NMR Spectrum of Compound $\mathbf{2 h}\left(400 \mathrm{MHz}, \mathrm{CDCl}_{3}\right)$

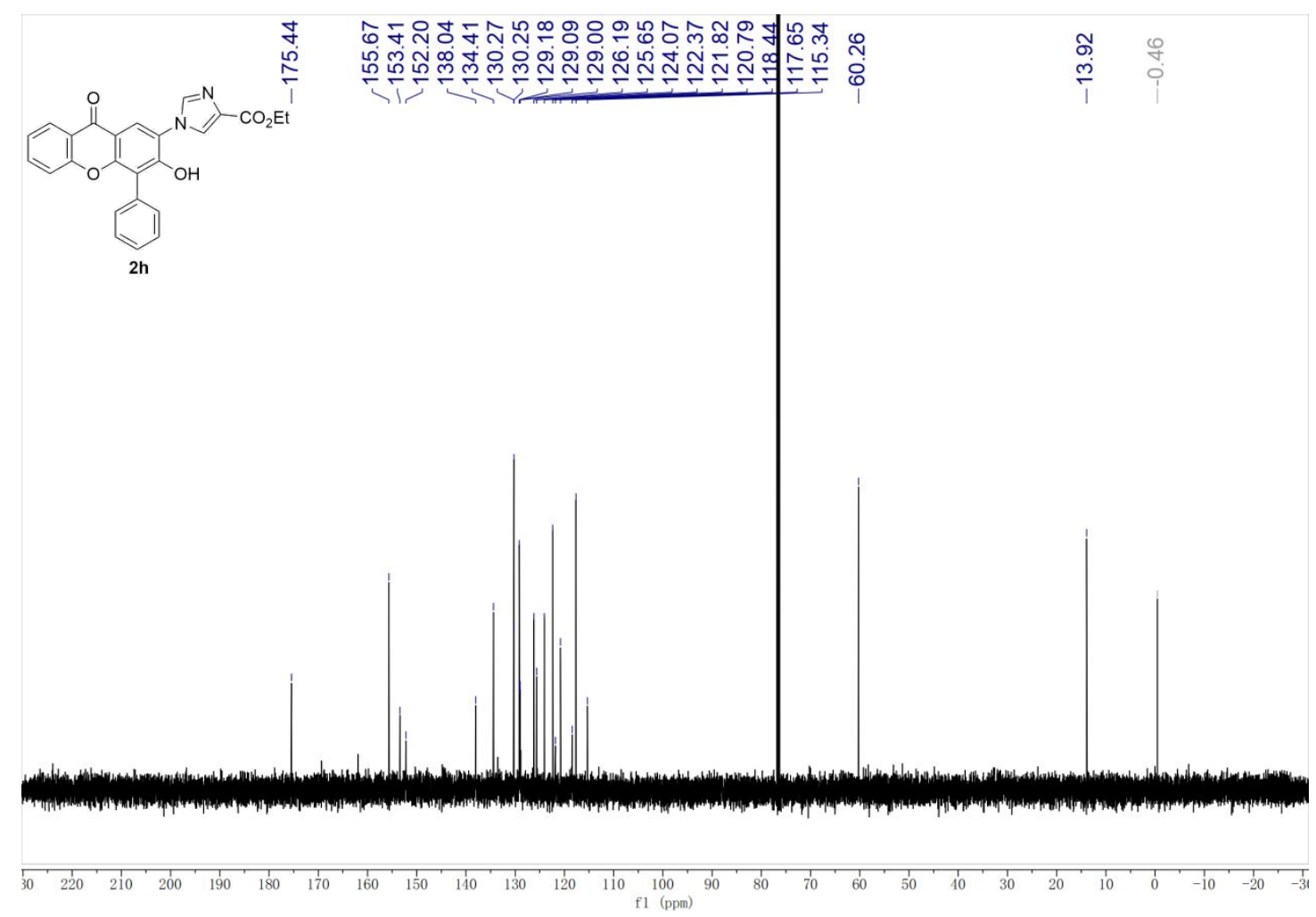

Figure S21: ${ }^{13} \mathrm{C}$ NMR Spectrum of Compound $\mathbf{2 h}\left(125 \mathrm{MHz}, \mathrm{CDCl}_{3}\right)$ 


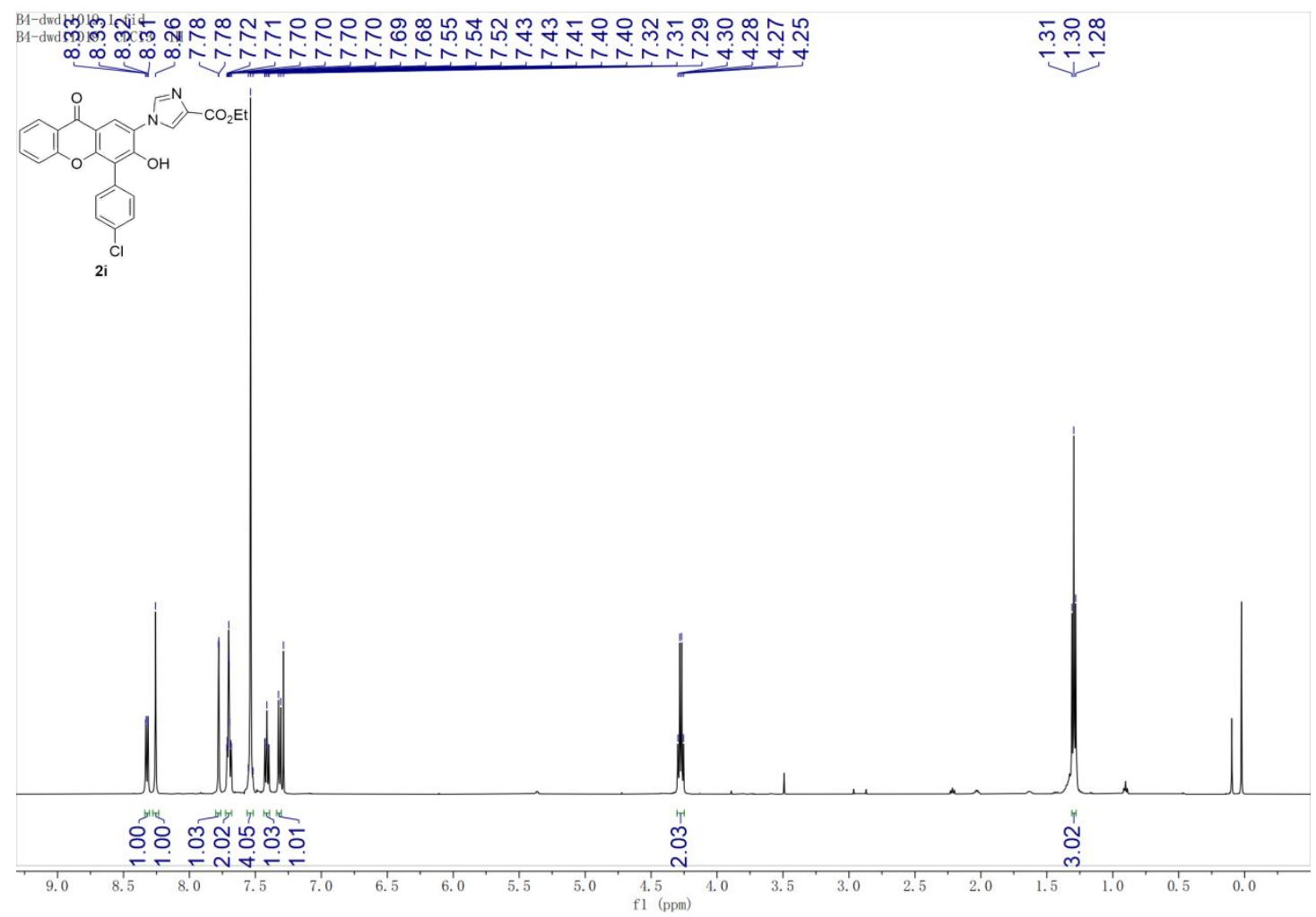

Figure S22: ${ }^{1} \mathrm{H}$ NMR Spectrum of Compound $2 \mathrm{i}\left(500 \mathrm{MHz}, \mathrm{CDCl}_{3}\right)$

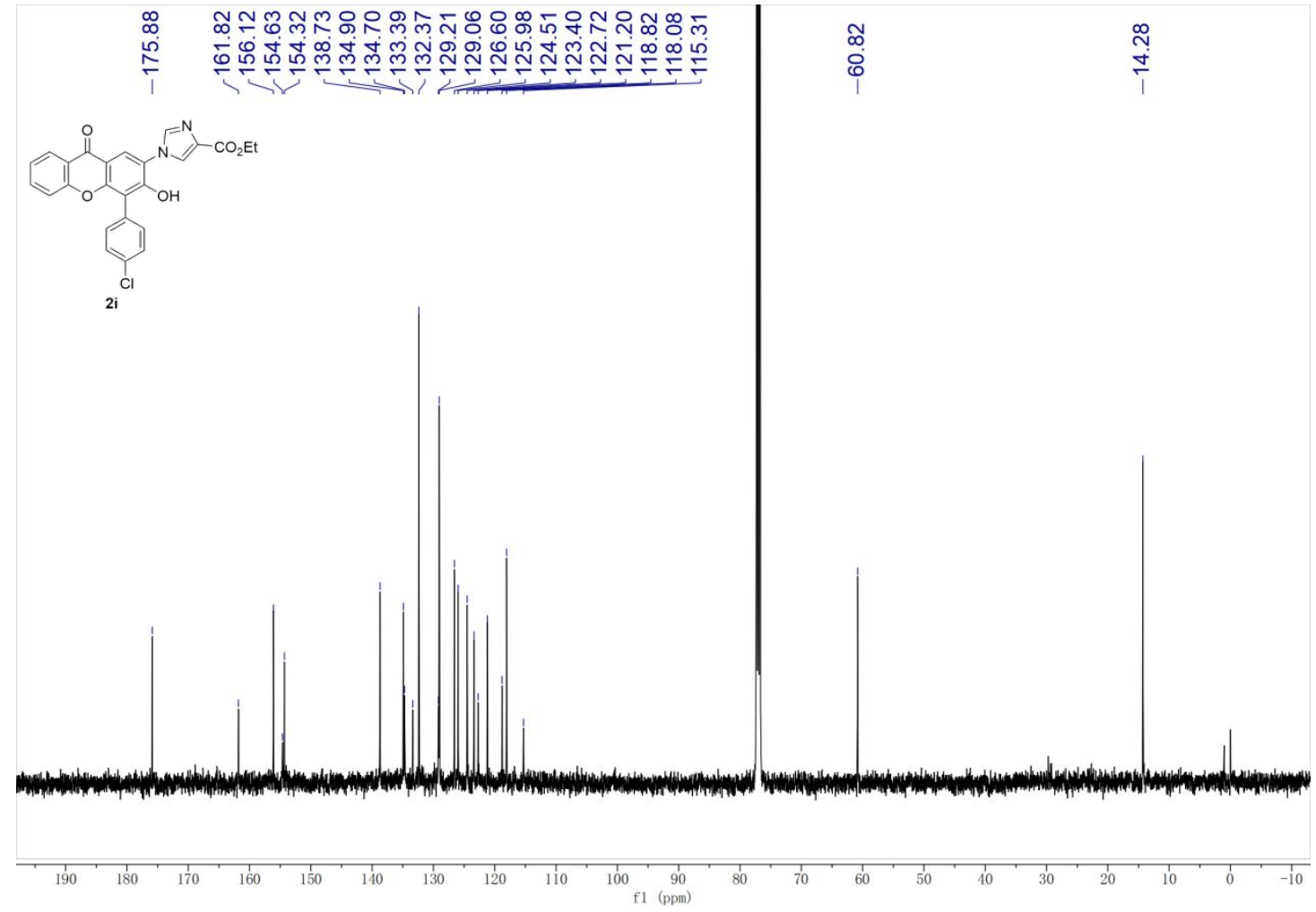

Figure S23: ${ }^{13} \mathrm{C}$ NMR Spectrum of Compound $2 \mathbf{i}\left(125 \mathrm{MHz}, \mathrm{CDCl}_{3}\right)$ 


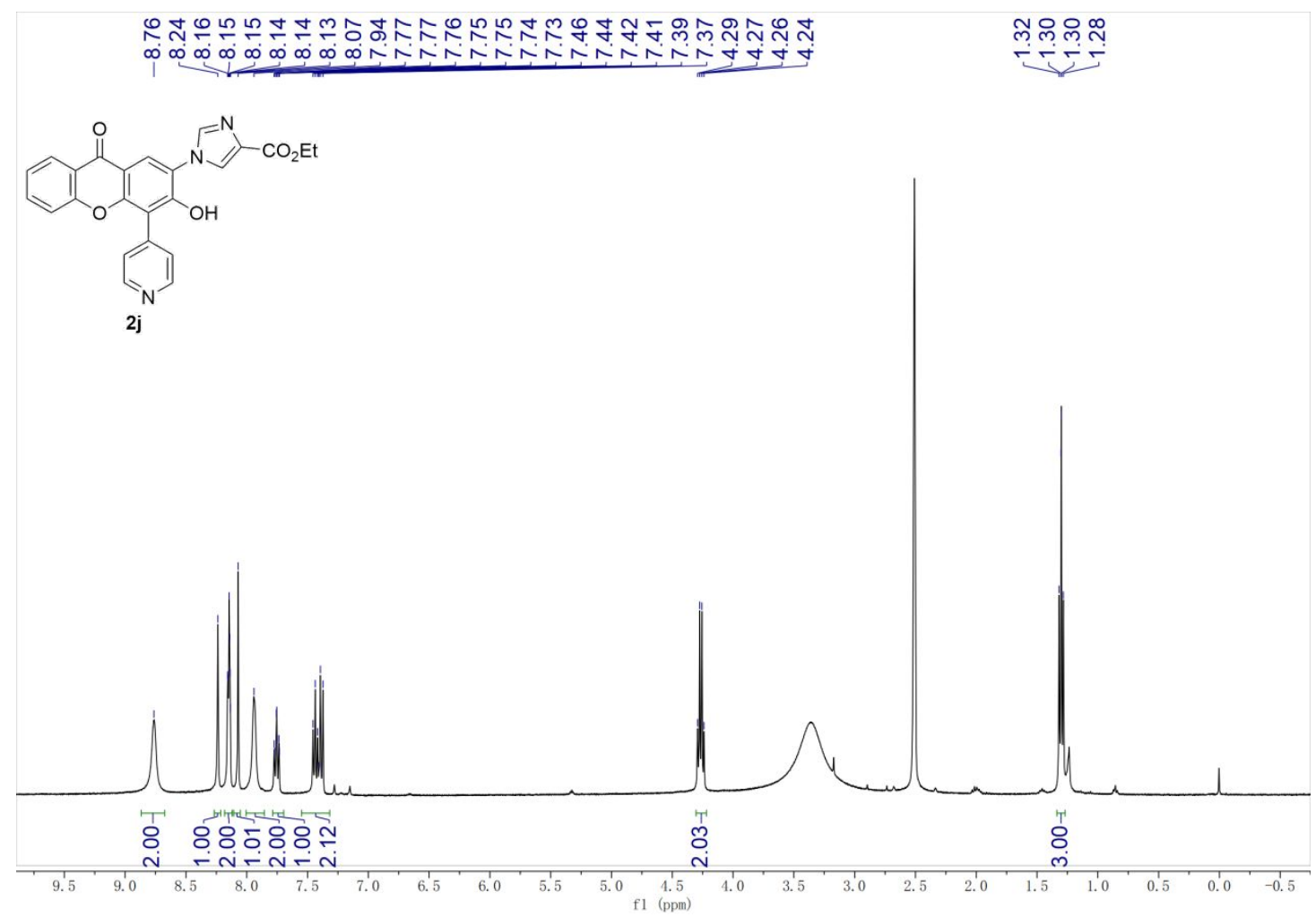

Figure S24: ${ }^{1} \mathrm{H}$ NMR Spectrum of Compound $2 \mathbf{j}\left(400 \mathrm{MHz}\right.$, DMSO- $\left.d_{6}\right)$

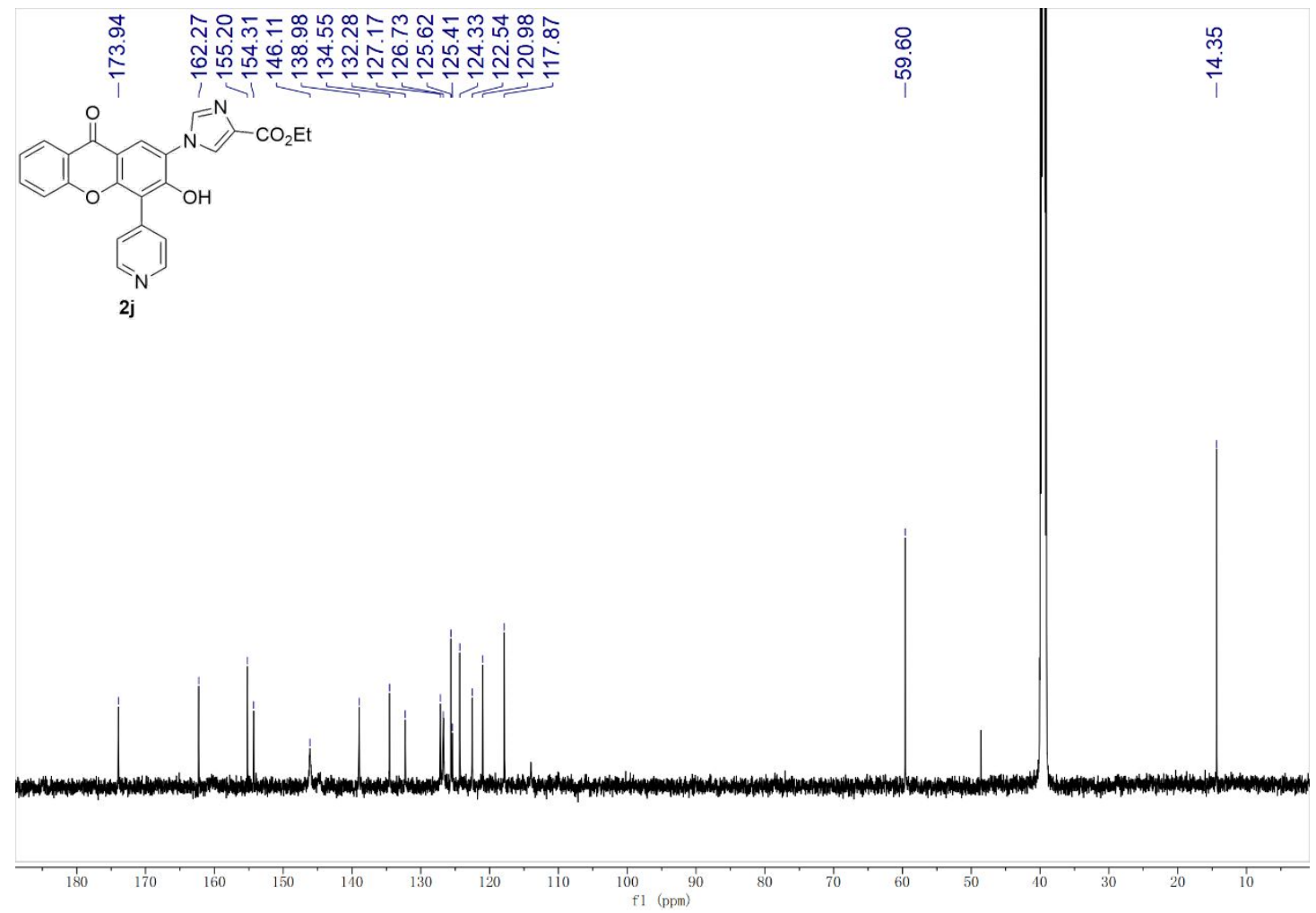

Figure S25: ${ }^{13} \mathrm{C}$ NMR Spectrum of Compound $\mathbf{2 j}\left(150 \mathrm{MHz}, 1: 20 \mathrm{v} / \mathrm{v} \mathrm{CD}_{3} \mathrm{OD}\right.$ in DMSO- $\left.d_{6}\right)$ 


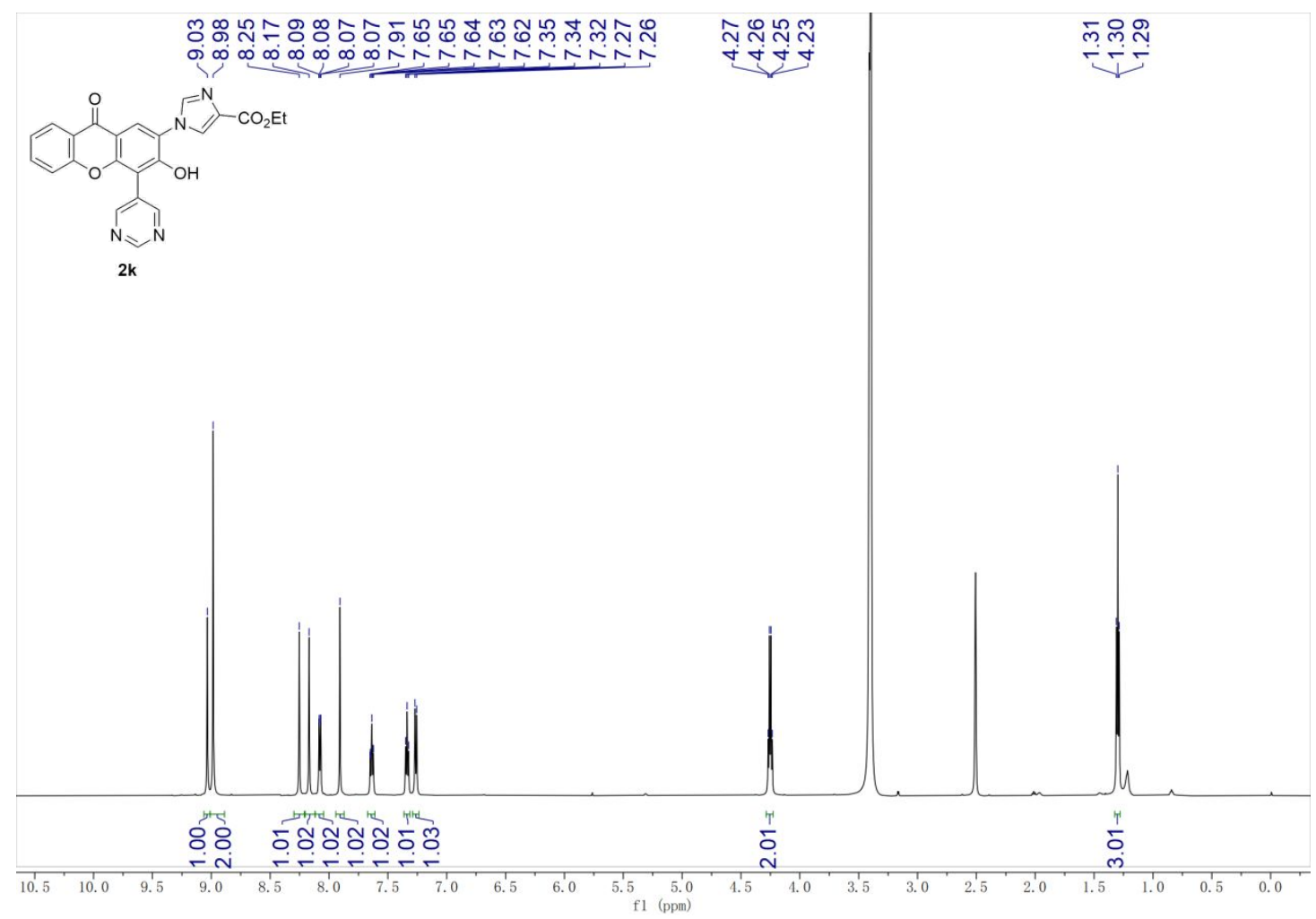

Figure S26: ${ }^{1} \mathrm{H}$ NMR Spectrum of Compound $2 \mathbf{k}\left(600 \mathrm{MHz}\right.$, DMSO- $\left.d_{6}\right)$

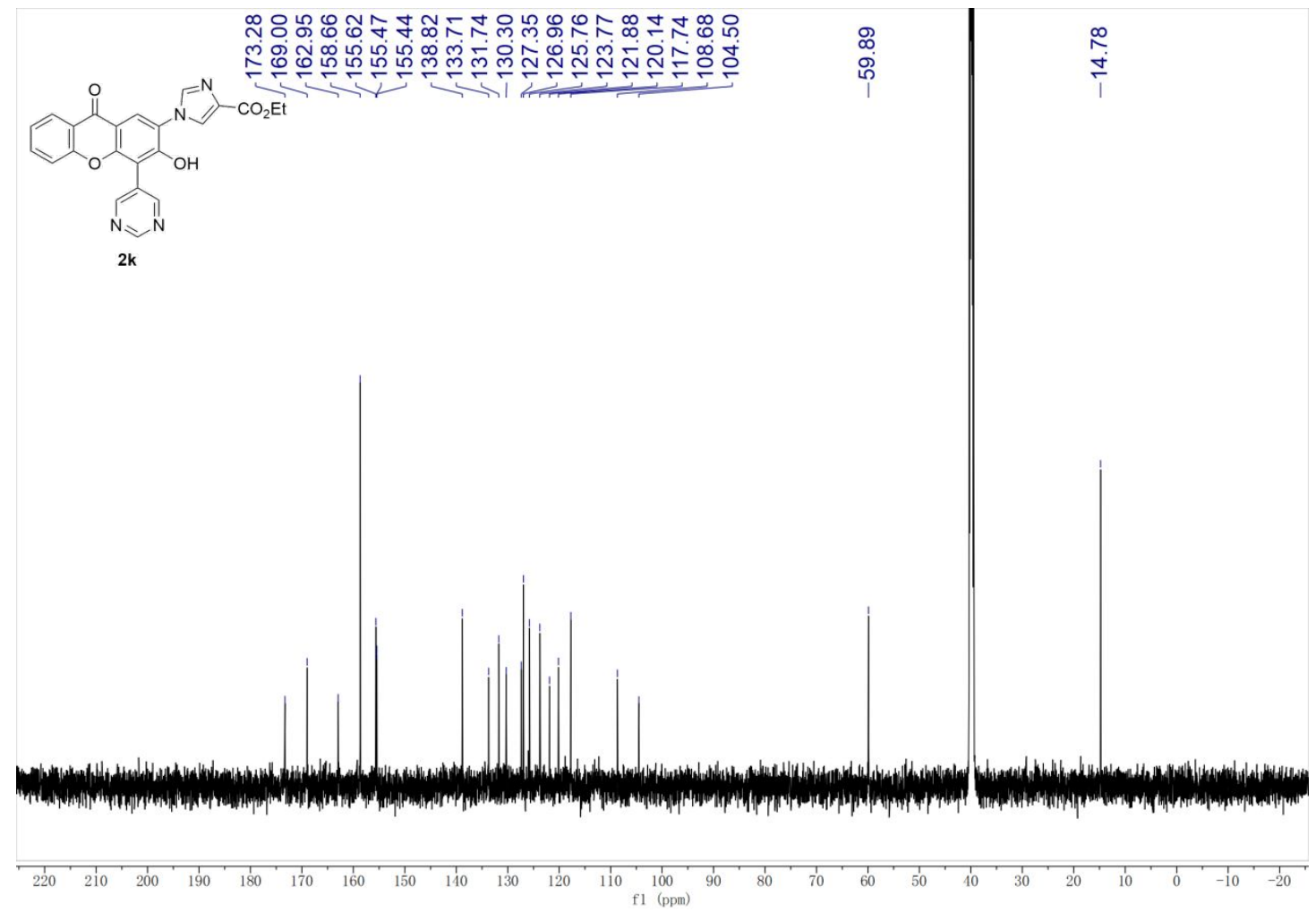

Figure S27: ${ }^{13} \mathrm{C}$ NMR Spectrum of Compound $2 \mathbf{k}\left(150 \mathrm{MHz}\right.$, DMSO- $\left.d_{6}\right)$ 


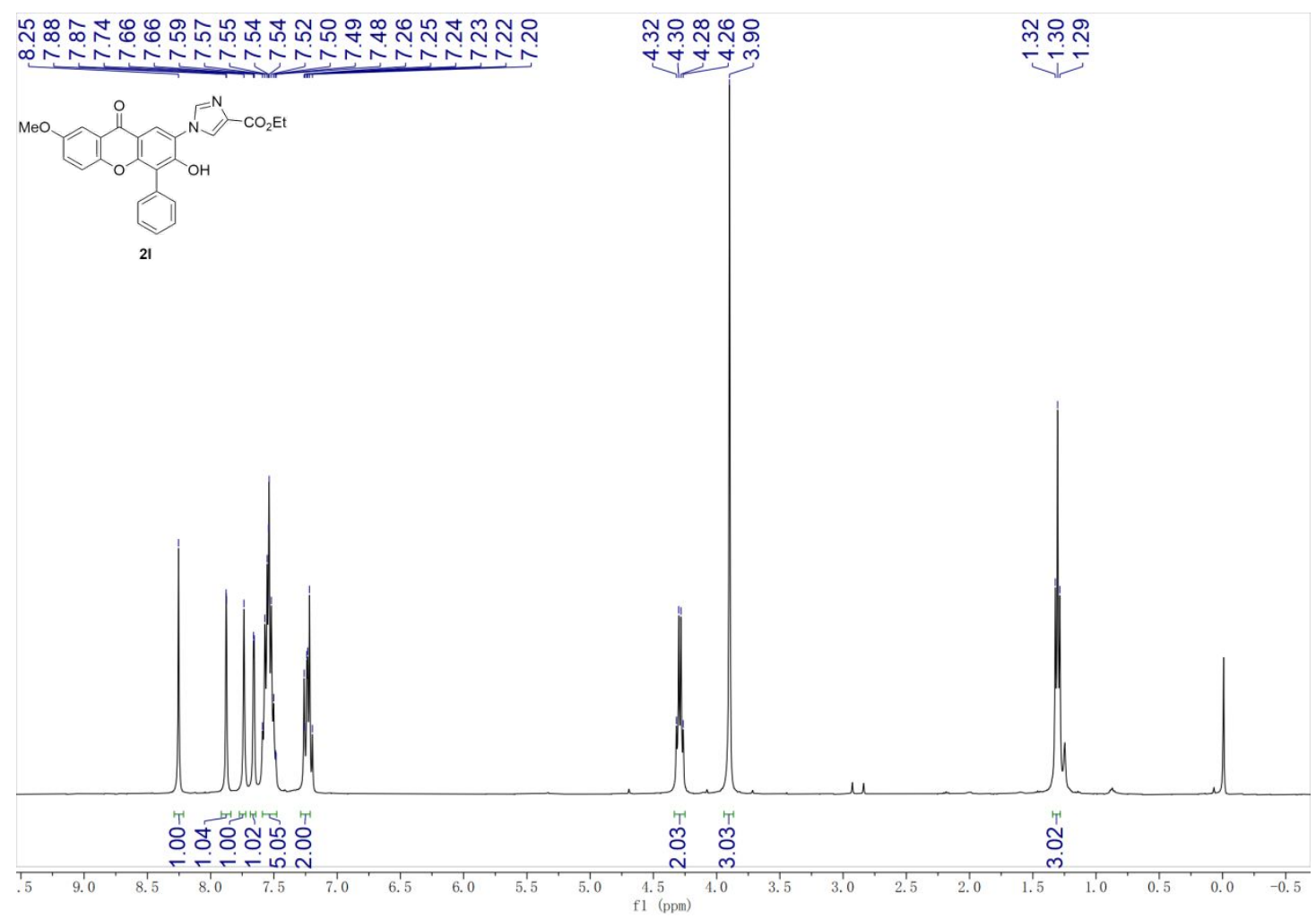

Figure S28: ${ }^{1} \mathrm{H}$ NMR Spectrum of Compound $21\left(400 \mathrm{MHz}, \mathrm{CDCl}_{3}\right)$
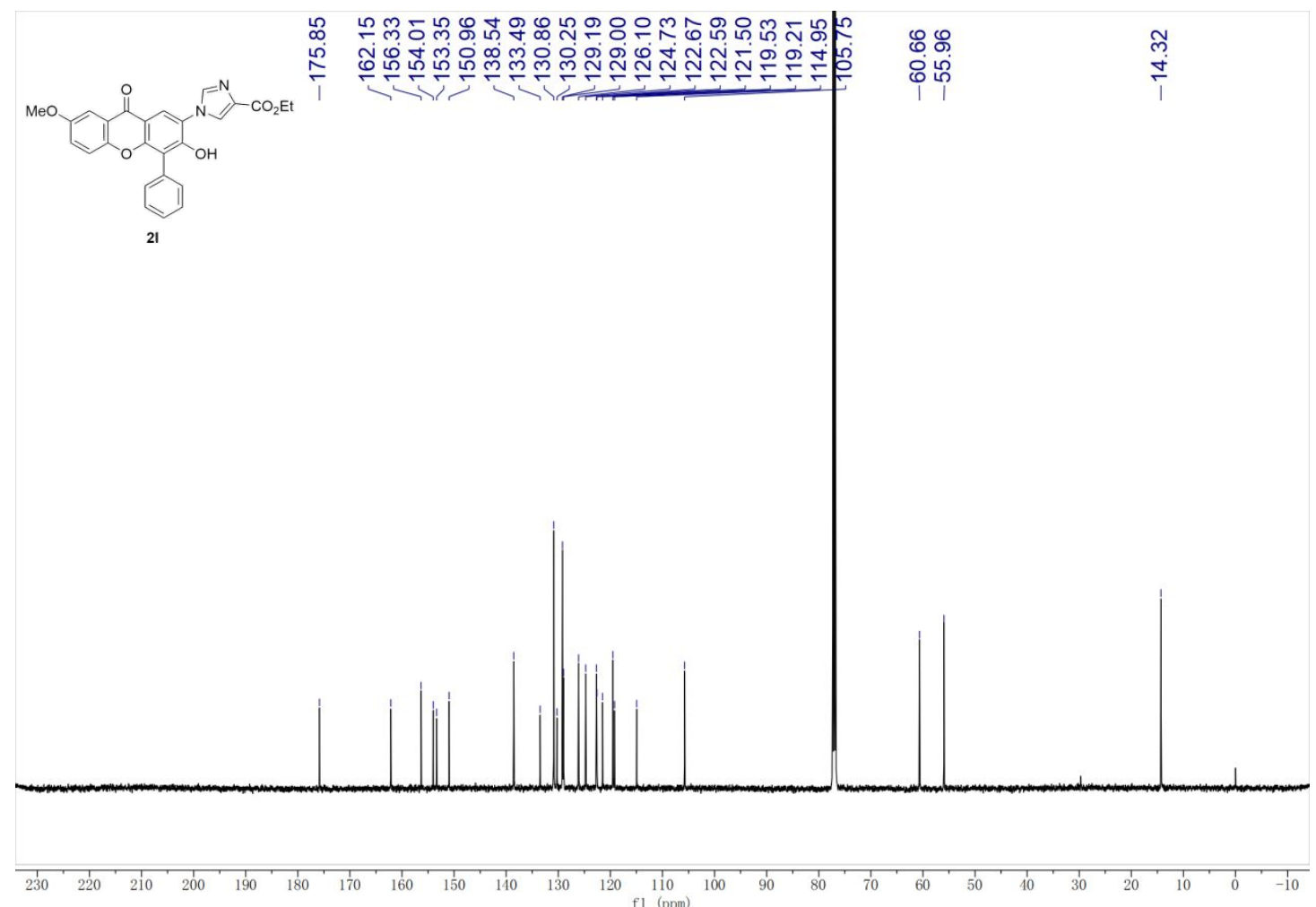

Figure S29: ${ }^{13} \mathrm{C}$ NMR Spectrum of Compound $2 \mathbf{l}\left(125 \mathrm{MHz}, \mathrm{CDCl}_{3}\right)$ 


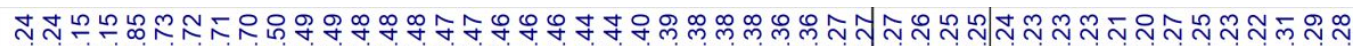

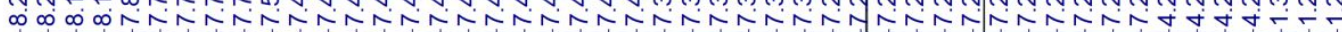<smiles>CCOc1cn(-c2cc3c(=O)c4cc(F)ccc4oc3c(-c3ccccc3)c2O)cn1</smiles>

$2 \mathrm{~m}$

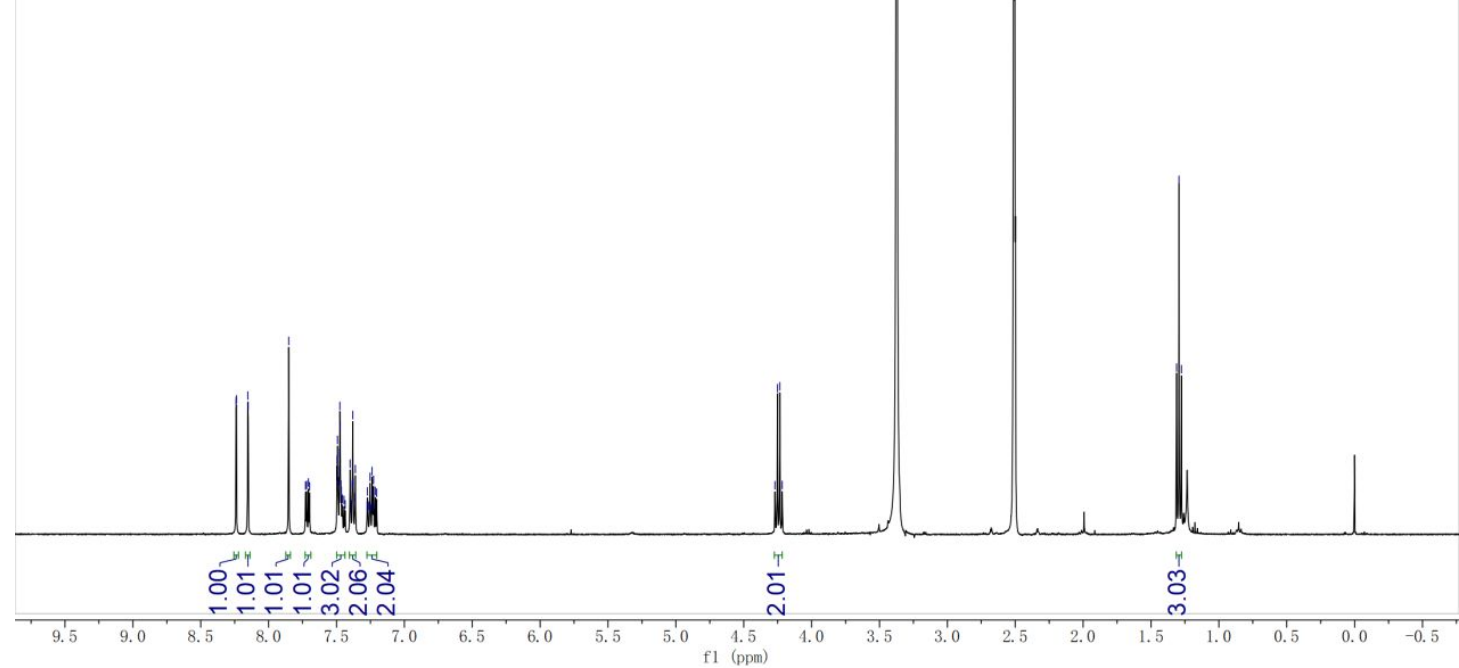

Figure S30: ${ }^{1} \mathrm{H}$ NMR Spectrum of Compound $\mathbf{2 m}\left(400 \mathrm{MHz}\right.$, DMSO- $\left.d_{6}\right)$

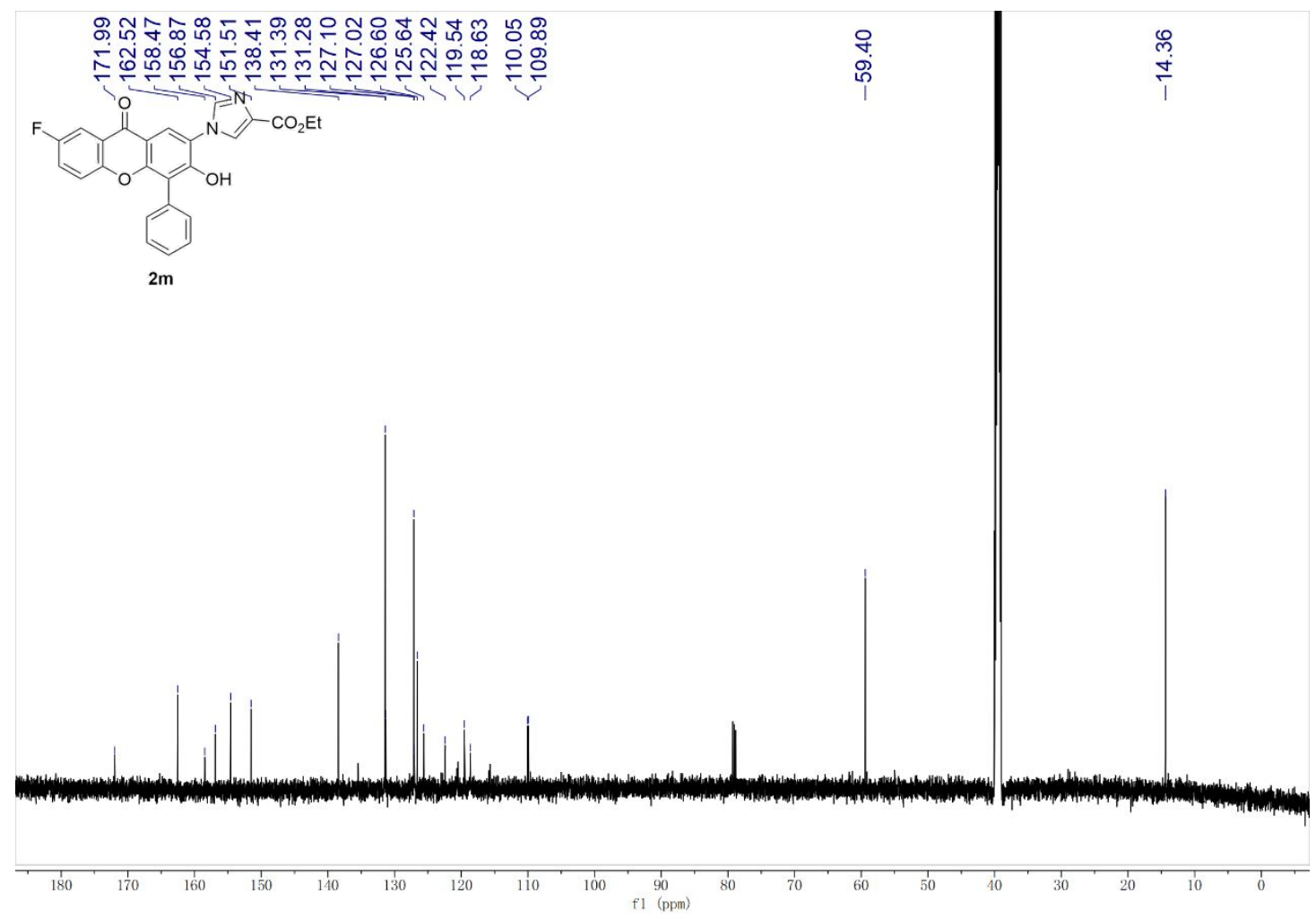

Figure S31: ${ }^{13} \mathrm{C}$ NMR Spectrum of Compound $2 \mathbf{m}\left(150 \mathrm{MHz}\right.$, DMSO- $\left.d_{6}\right)$ 


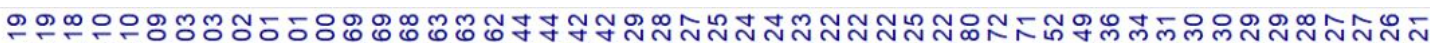
চ<smiles>CCOC(=O)c1cn(-c2cc3c(=O)c4ccccc4oc3c(C3CCCCC3)c2O)cn1</smiles>

$2 n$

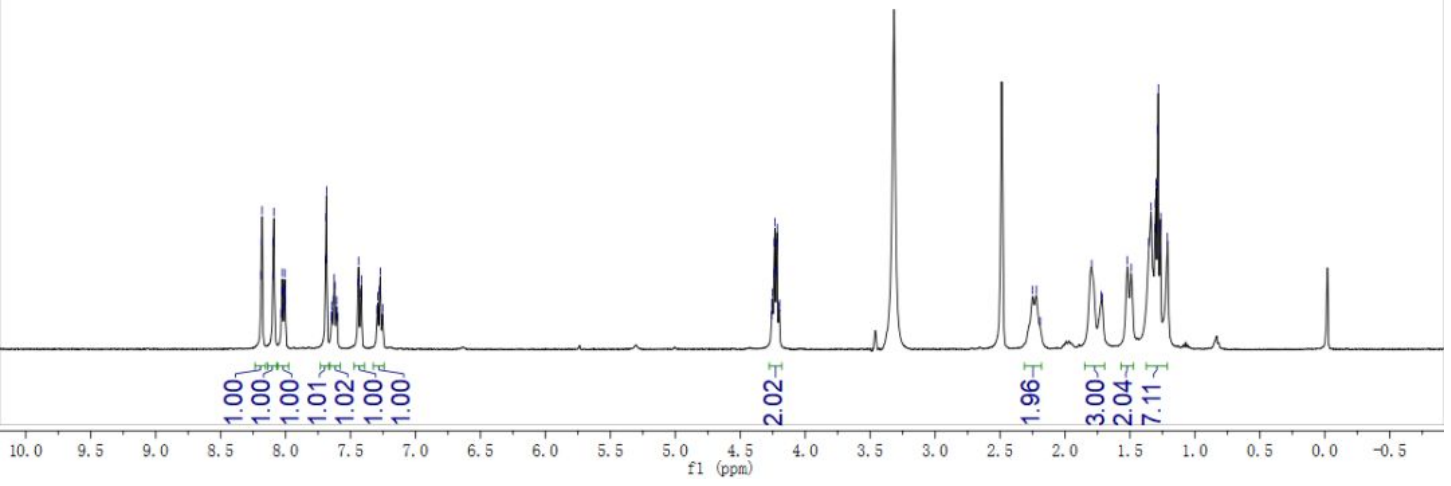

Figure S32: ${ }^{1} \mathrm{H}$ NMR Spectrum of Compound 2n(400 MHz, DMSO- $\left.d_{6}\right)$

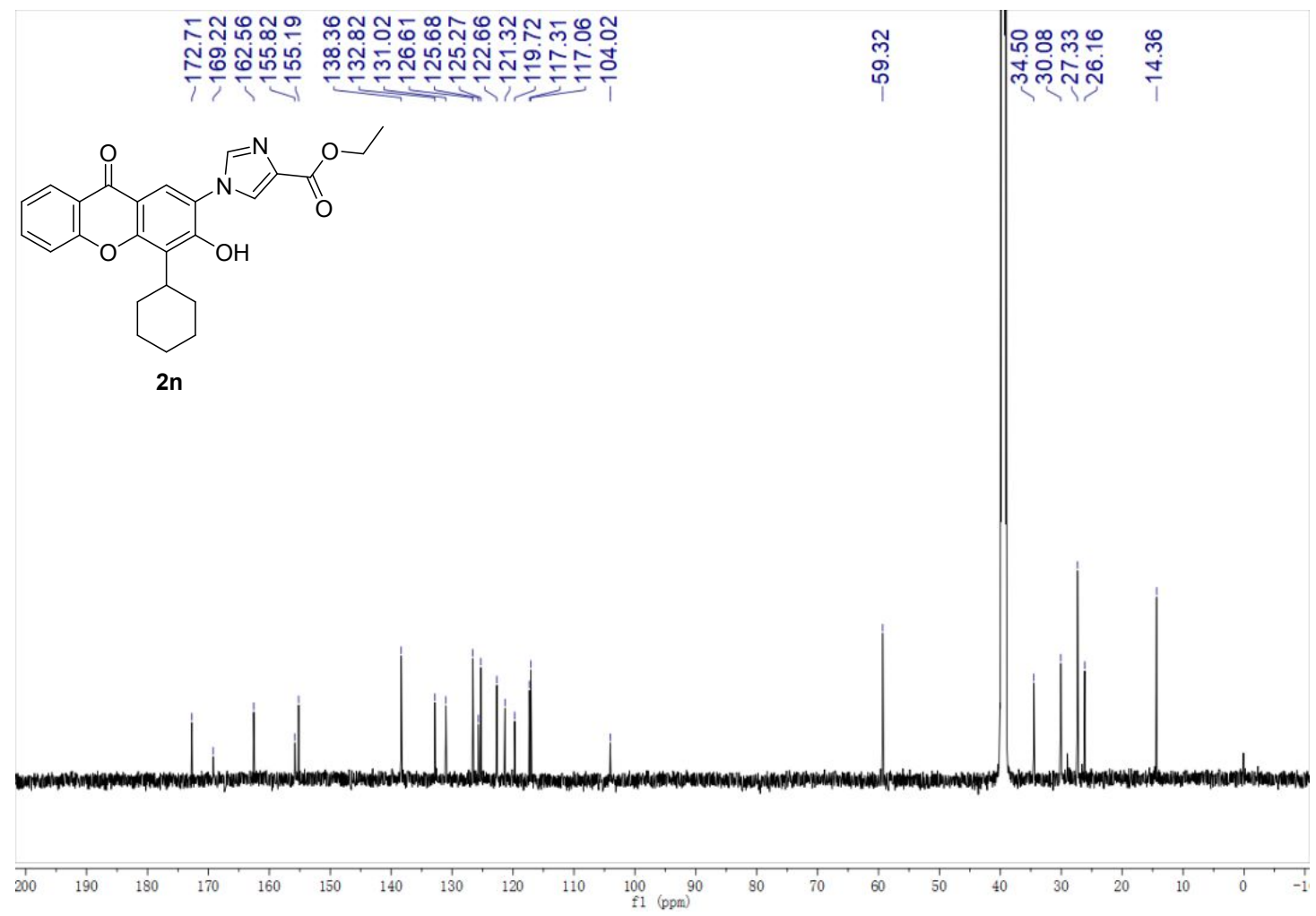

Figure S33: ${ }^{13} \mathrm{C}$ NMR Spectrum of Compound 2n(150 MHz, DMSO- $\left.d_{6}\right)$ 


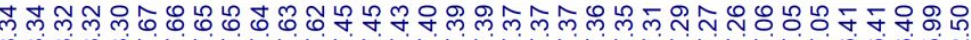

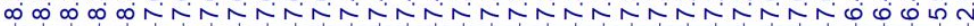

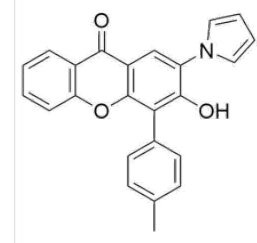

$4 a$

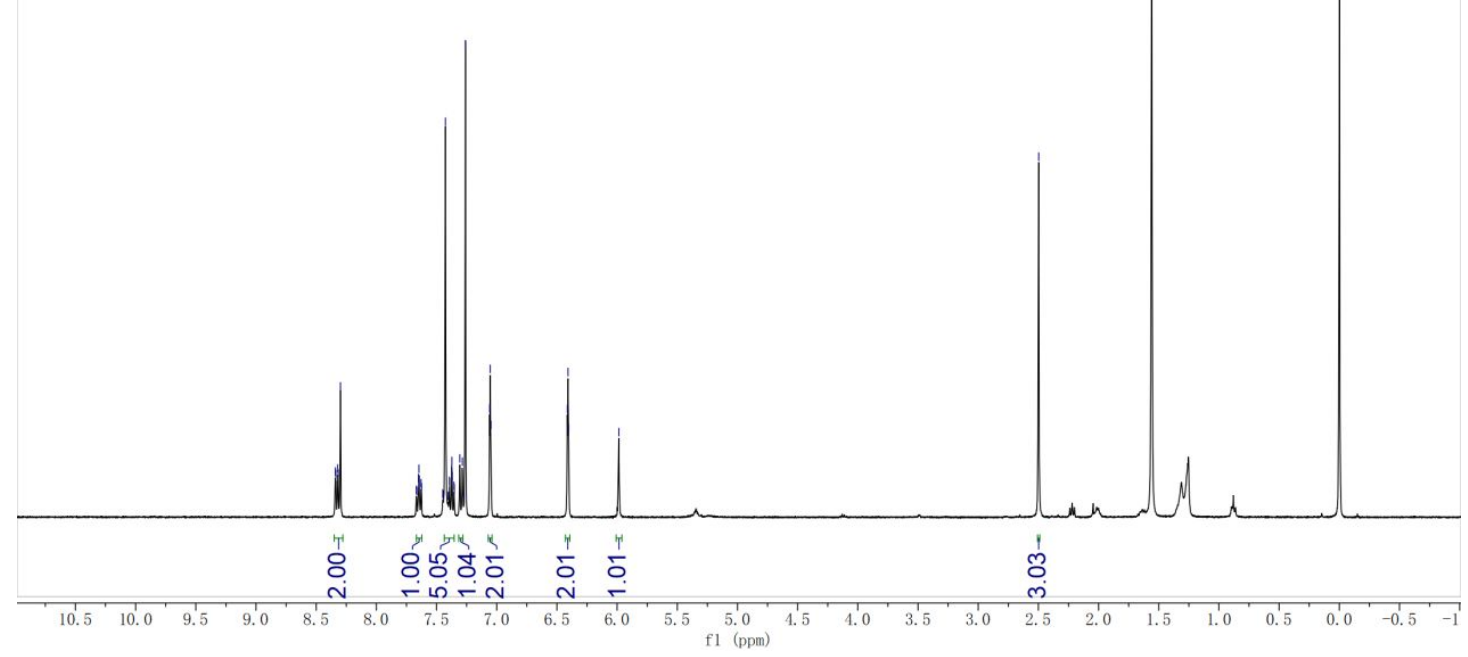

Figure S34: ${ }^{1} \mathrm{H}$ NMR Spectrum of Compound $4 \mathrm{a}\left(500 \mathrm{MHz}, \mathrm{CDCl}_{3}\right)$

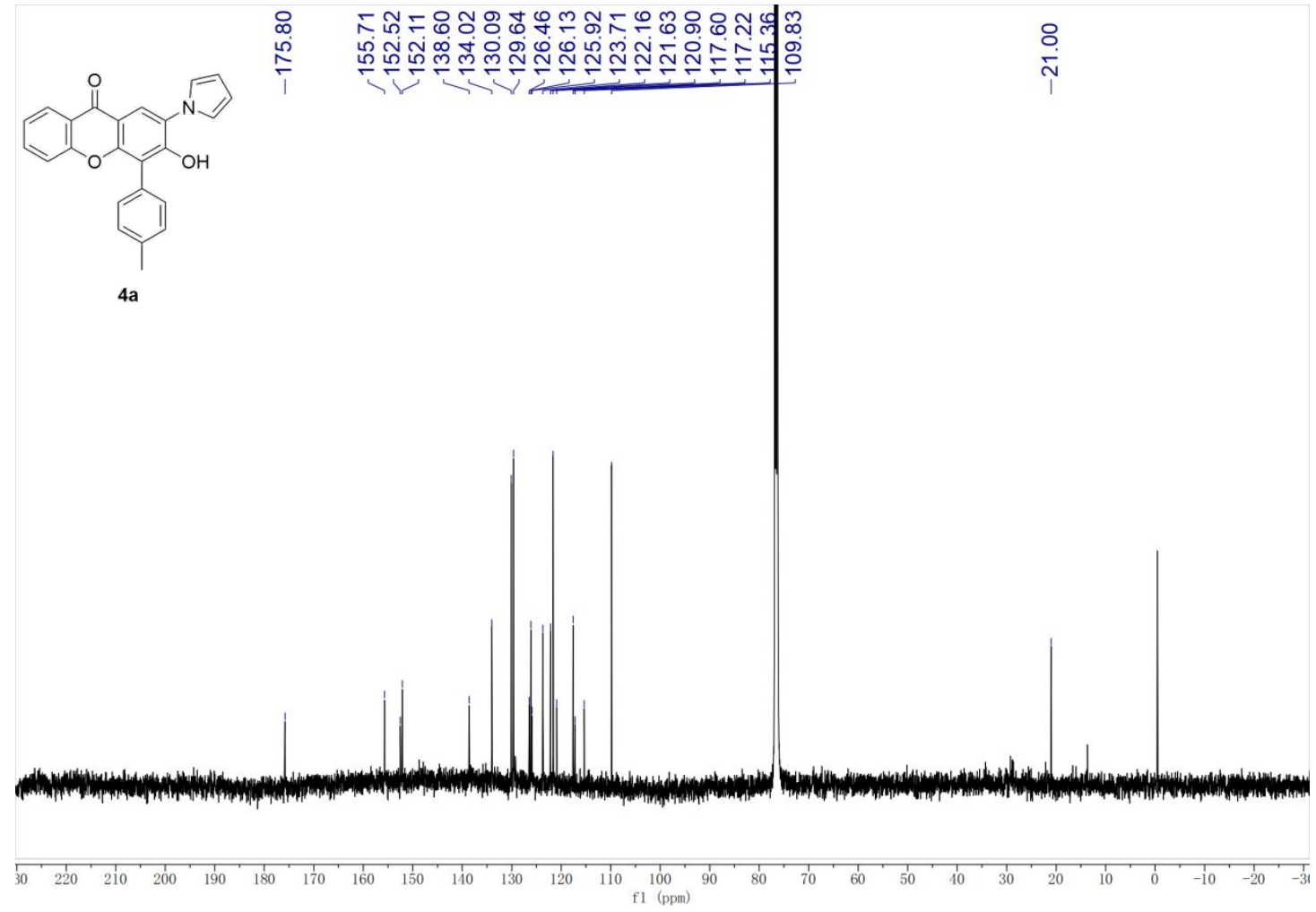

Figure S35: ${ }^{13} \mathrm{C}$ NMR Spectrum of Compound $4 \mathbf{a}\left(125 \mathrm{MHz}, \mathrm{CDCl}_{3}\right)$ 

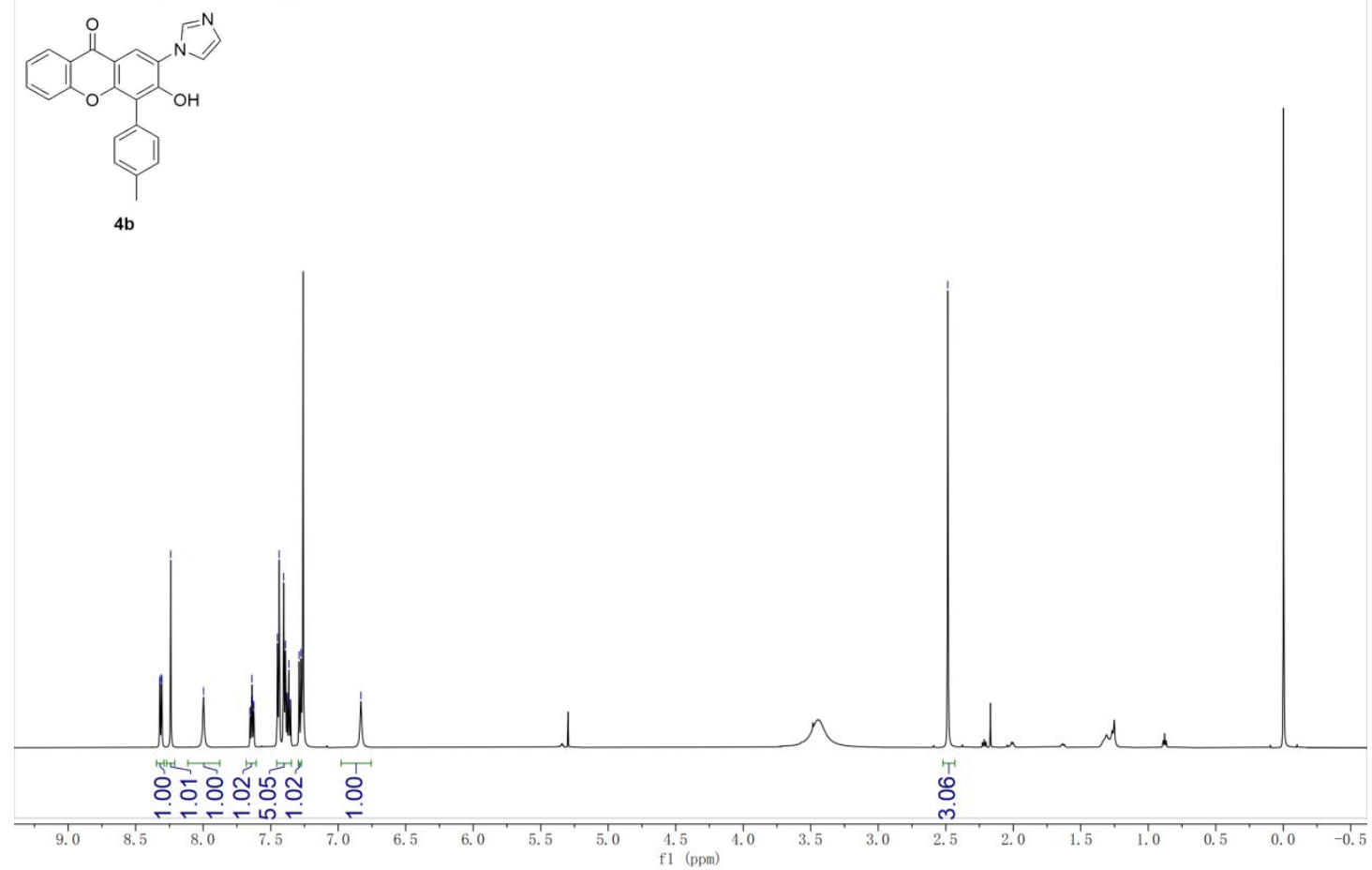

Figure S36: ${ }^{1} \mathrm{H}$ NMR Spectrum of Compound $\mathbf{4 b}\left(600 \mathrm{MHz}, \mathrm{CDCl}_{3}\right)$

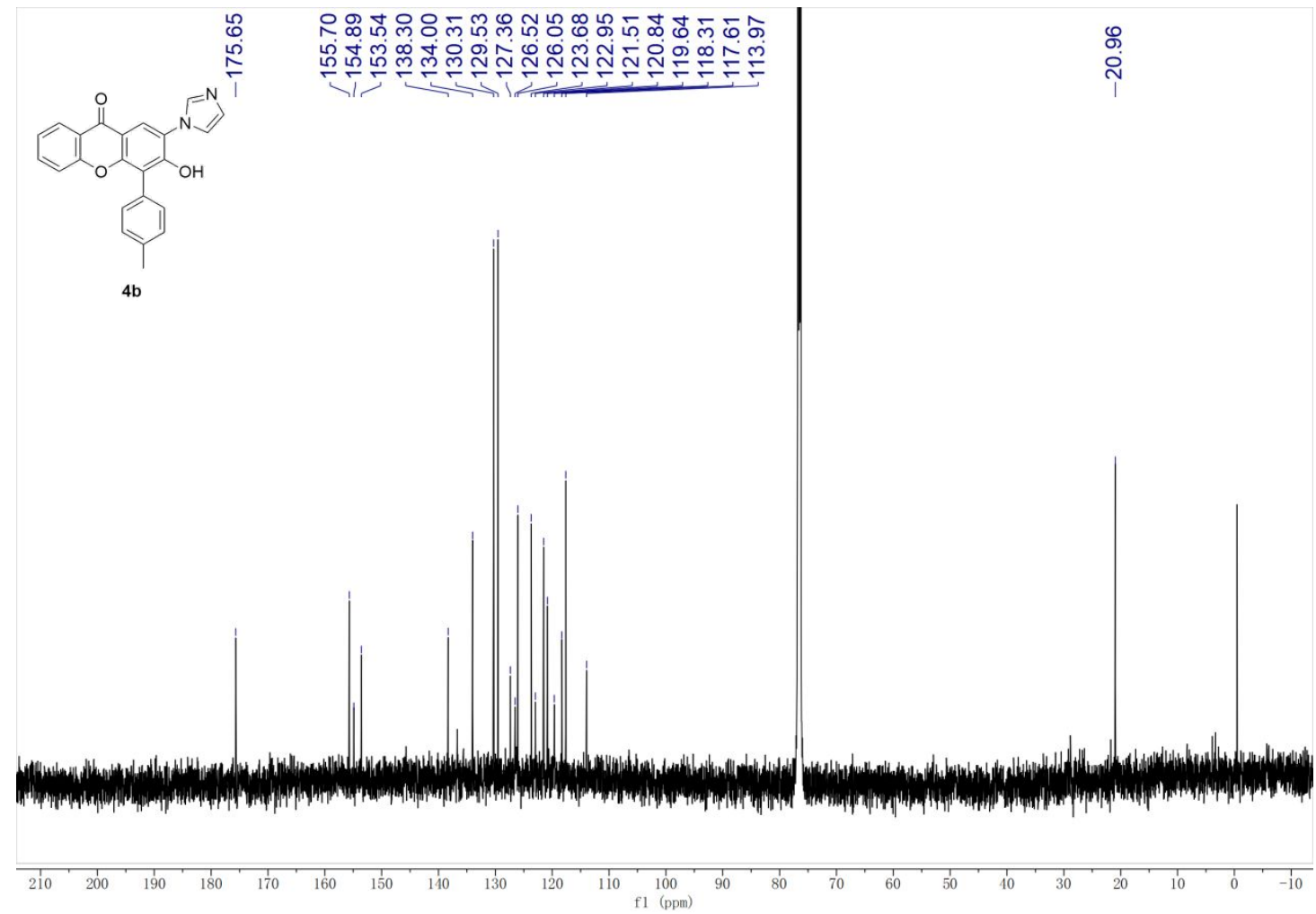

Figure S37: ${ }^{13} \mathrm{C}$ NMR Spectrum of Compound $4 \mathbf{b}\left(150 \mathrm{MHz}, \mathrm{CDCl}_{3}\right)$ 


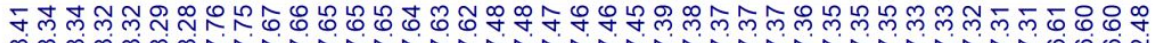

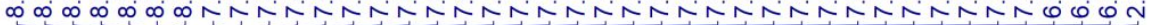

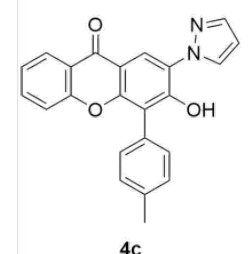

$4 c$
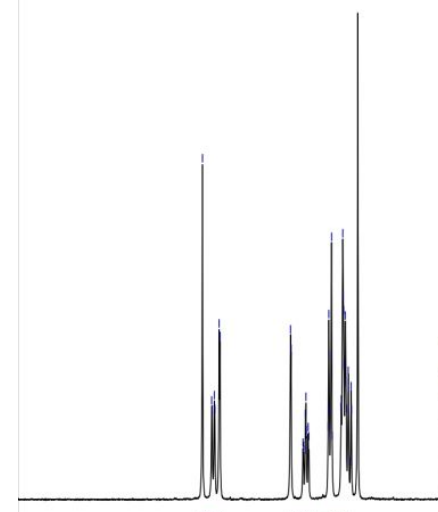

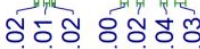

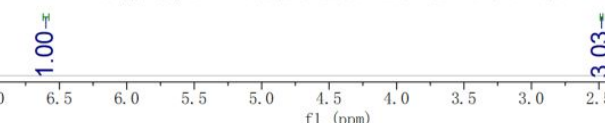

ஜे

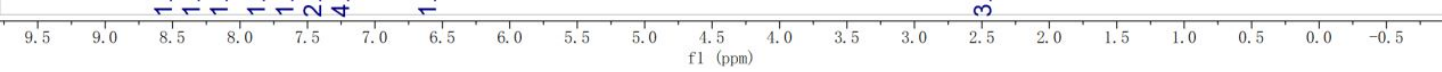

Figure S38: ${ }^{1} \mathrm{H}$ NMR Spectrum of Compound $4 \mathbf{c}\left(400 \mathrm{MHz}, \mathrm{CDCl}_{3}\right)$

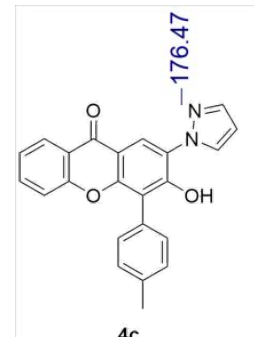

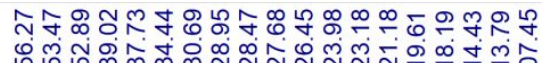

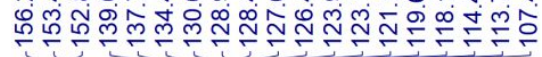

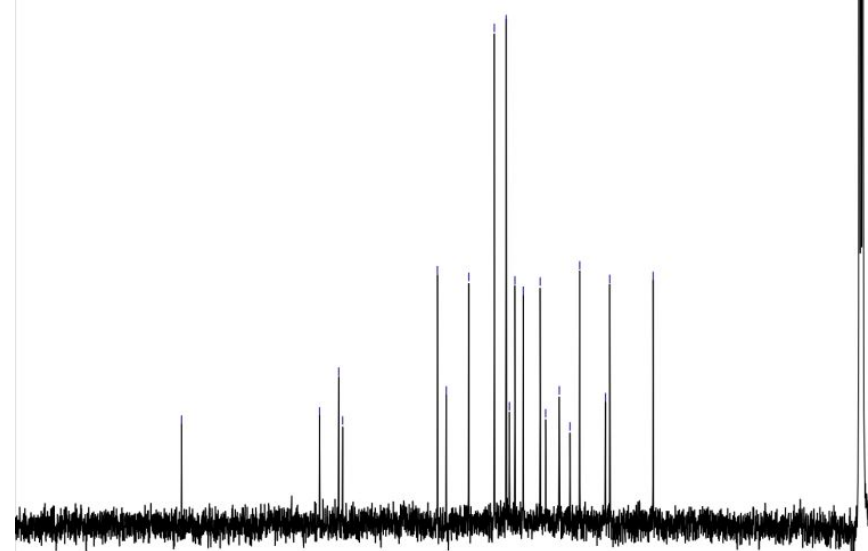

00

$(\mathrm{ppm})$

Figure S39: ${ }^{13} \mathrm{C}$ NMR Spectrum of Compound $4 \mathbf{c}\left(125 \mathrm{MHz}, \mathrm{CDCl}_{3}\right)$ 
3. X-ray Crystallography data of compound $\mathbf{2 i}$

Compound $2 \mathbf{i}$ was recrystallized in mixed solvent of hexane and dichloromethane.

CCDC 1987456 (2i) contains the supplementary crystallographic data for this paper. These data can be obtained free of charge from The Cambridge Crystallographic Data Centre via $\underline{\text { www.ccdc.cam.ac.uk/data request/cif }}$

Table S2 Crystal refinement data of compound $\mathbf{2} \mathbf{i}$

\begin{tabular}{|c|c|}
\hline Empirical formula & $\mathrm{C}_{28} \mathrm{H}_{24} \mathrm{ClN}_{2} \mathrm{O}_{5}$ \\
\hline Formula weight & 503.94 \\
\hline Temperature/K & 110.0 \\
\hline Crystal system & tetragonal \\
\hline Space group & $\mathrm{P} 4 / \mathrm{n}$ \\
\hline $\mathrm{a} / \AA \AA$ & $26.429(2)$ \\
\hline $\mathrm{b} / \AA$ & $26.429(2)$ \\
\hline $\mathrm{c} / \AA \AA$ & $7.8670(7)$ \\
\hline$\alpha /^{\circ}$ & 90 \\
\hline$\beta /^{\circ}$ & 90 \\
\hline$\gamma /{ }^{\circ}$ & 90 \\
\hline Volume $/ \AA^{3}$ & $5495.1(11)$ \\
\hline $\mathrm{Z}$ & 8 \\
\hline$\rho_{\text {calc }} \mathrm{g} / \mathrm{cm}^{3}$ & 1.218 \\
\hline$\mu / \mathrm{mm}^{-1}$ & 0.177 \\
\hline $\mathrm{F}(000)$ & 2104.0 \\
\hline Crystal size $/ \mathrm{mm}^{3}$ & $0.12 \times 0.08 \times 0.05$ \\
\hline Radiation & $\operatorname{MoK} \alpha(\lambda=0.71073)$ \\
\hline $2 \Theta$ range for data collection ${ }^{\circ}$ & 4.36 to 50.018 \\
\hline Index ranges & $-31 \leq \mathrm{h} \leq 31,-28 \leq \mathrm{k} \leq 27,-9 \leq 1 \leq 8$ \\
\hline Reflections collected & 18173 \\
\hline Independent reflections & $4844\left[\mathrm{R}_{\mathrm{int}}=0.1201, \mathrm{R}_{\text {sigma }}=0.1197\right]$ \\
\hline Data/restraints/parameters & $4844 / 0 / 328$ \\
\hline Goodness-of-fit on $\mathrm{F}^{2}$ & 1.048 \\
\hline Final $R$ indexes $[I>=2 \sigma(I)]$ & $\mathrm{R}_{1}=0.0746, \mathrm{wR}_{2}=0.1877$ \\
\hline Final $\mathrm{R}$ indexes [all data] & $\mathrm{R}_{1}=0.1561, \mathrm{wR}_{2}=0.2393$ \\
\hline Largest diff. peak/hole / e $\AA^{-3}$ & $0.57 /-0.41$ \\
\hline
\end{tabular}

